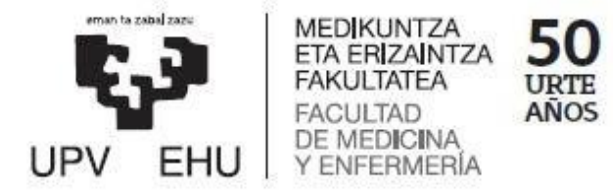

\title{
Repercusiones del consumo de cannabis en la evolución del trastorno bipolar
}




\section{CONTENIDO}

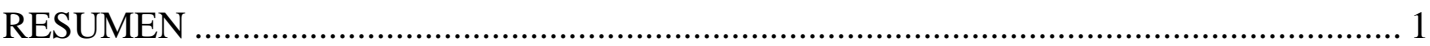

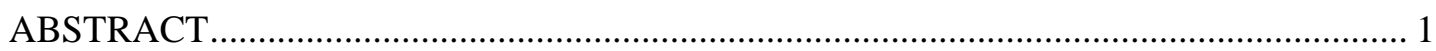

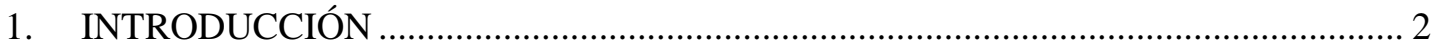

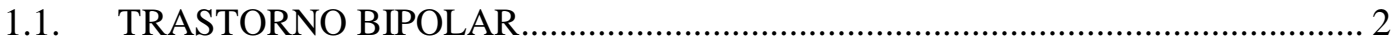

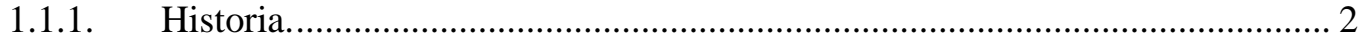

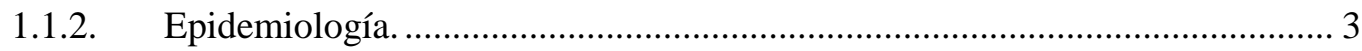

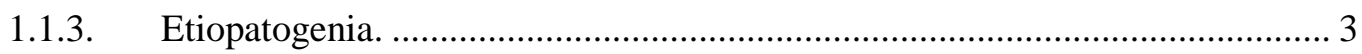

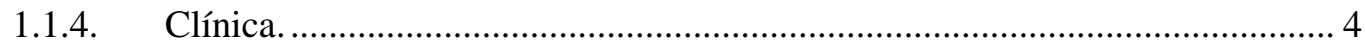

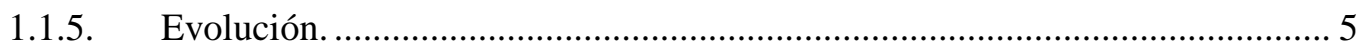

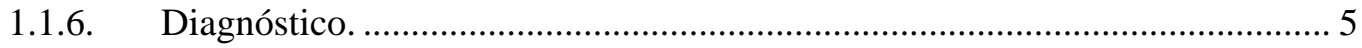

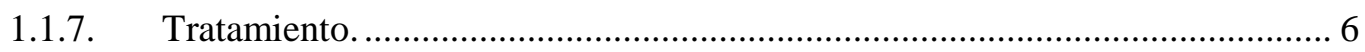

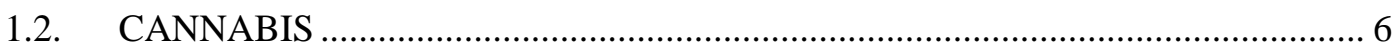

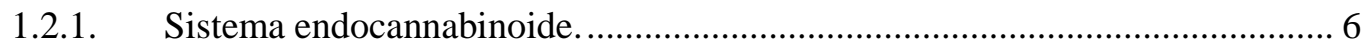

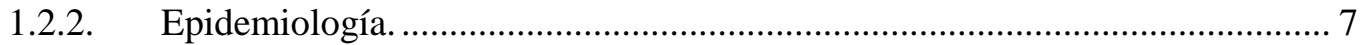

1.2.3. Características clínicas del consumo de cannabis............................................ 7

1.2.4. Tratamiento de la dependencia de cannabis................................................... 8

1.3. CONSUMO DE DROGAS Y TRASTORNO BIPOLAR …................................... 8

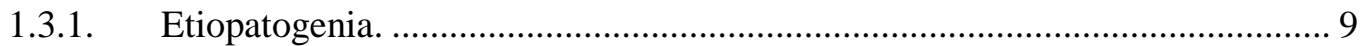

1.3.2. Efecto del trastorno por uso de sustancias comórbido sobre la clínica y el curso



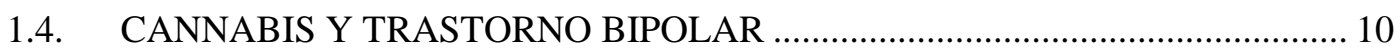

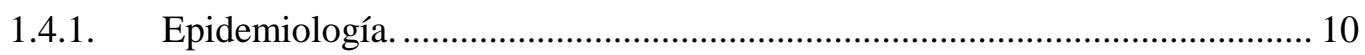

1.4.2. Dependencia de cannabis y trastorno bipolar................................................ 10

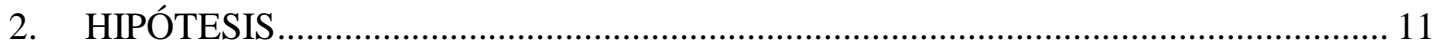

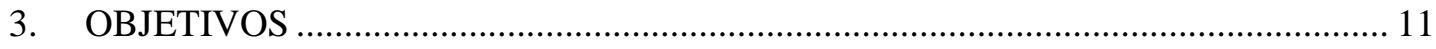

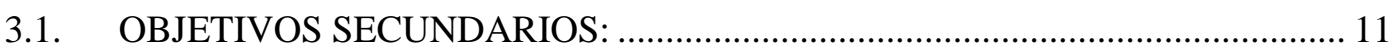




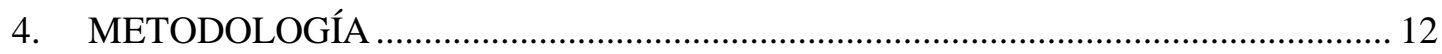

4.1. CRITERIOS DE SELECCIÓN DE ARTÍCULOS ................................................ 13

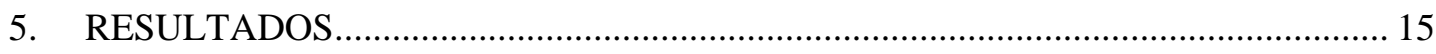

5.1. REPERCUSIÓN DEL CONSUMO DE CANNABIS EN LA EVOLUCIÓN DEL

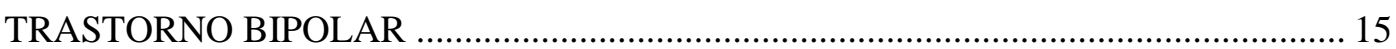

5.2. CARACTERÍSTICAS BASALES DIFERENCIALES ENTRE LOS

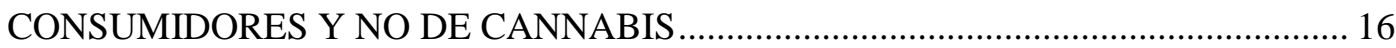

5.3. INFLUENCIA DE LAS CARACTERÍSTICAS SOCIODEMOGRÁFICAS EN EL EFECTO DEL CONSUMO SOBRE LA EVOLUCIÓN DEL TRASTORNO BIPOLAR17

5.4. INFLUENCIA DE LAS CARACTERÍSTICAS DEL TRASTORNO BIPOLAR EN LOS EFECTOS DEL CONSUMO DE CANNABIS EN LA EVOLUCIÓN

5.5. INFLUENCIA DE LAS CARACTERÍSTICAS DEL CONSUMO DE CANNABIS EN LOS EFECTOS DEL CANNABIS EN LA EVOLUCIÓN

5.6. EFECTOS DEL POLICONSUMO EN LOS EFECTOS DEL CONSUMO DE CANNABIS SOBRE EL TRASTORNO BIPOLAR 20

5.7. EFECTOS DEL CESE DEL CONSUMO EN LA EVOLUCIÓN DEL TRASTORNO BIPOLAR

5.8. EFECTOS COGNITIVOS DEL CONSUMO DE CANNABIS DURANTE EL SEGUIMIENTO DEL TRASTORNO BIPOLAR

5.9. EFECTOS DEL CONSUMO DE CANNABIS EN LA PRESENCIA DE CICLOS RÁPIDOS O DE CLÍNICA ATÍPICA

5.10. EFECTOS DEL CONSUMO DE CANNABIS EN LA RESISTENCIA AL TRATAMIENTO

6. DISCUSIÓN

6.1. REPERCUSIÓN DEL CONSUMO DE CANNABIS EN LA EVOLUCIÓN DEL TRASTORNO BIPOLAR 28

6.2. CARACTERÍSTICAS BASALES DIFERENCIALES ENTRE LOS CONSUMIDORES Y NO DE CANNABIS

6.3. INFLUENCIA DE LAS CARACTERÍSTICAS SOCIODEMOGRÁFICAS EN EL EFECTO DEL CONSUMO SOBRE LA EVOLUCIÓN DEL TRASTORNO BIPOLAR31 
6.4. INFLUENCIA DE LAS CARACTERÍSTICAS DEL TRASTORNO BIPOLAR EN LOS EFECTOS DEL CONSUMO DE CANNABIS EN LA EVOLUCIÓN 32

6.5. INFLUENCIA DE LAS CARACTERÍSTICAS DEL CONSUMO DE CANNABIS EN LOS EFECTOS DEL CANNABIS EN LA EVOLUCIÓN

6.6. EFECTOS DEL POLICONSUMO EN LOS EFECTOS DEL CONSUMO DE CANNABIS SOBRE EL TRASTORNO BIPOLAR 34

6.7. EFECTOS DEL CESE DEL CONSUMO EN LA EVOLUCIÓN DEL TRASTORNO BIPOLAR 36

6.8. EFECTOS COGNITIVOS DEL CONSUMO DE CANNABIS DURANTE EL SEGUIMIENTO DEL TRASTORNO BIPOLAR

6.9. EVALUAR LA REPERCUSIÓN DEL CONSUMO EN LA PRESENCIA DE CICLOS RÁPIDOS Y MANIFESTACIONES CLÍNICAS ATÍPICAS 37

6.10. EFECTOS DEL CONSUMO DE CANNABIS EN LA RESISTENCIA AL TRATAMIENTO 38

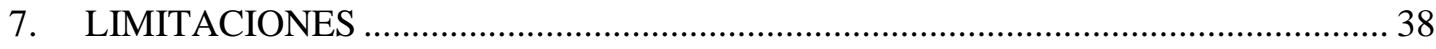

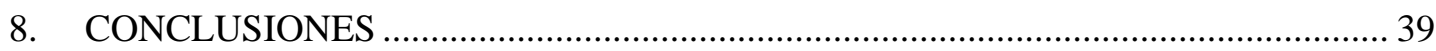

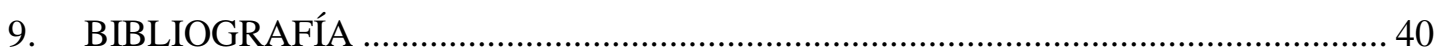

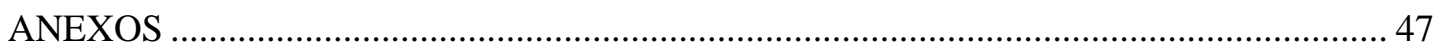

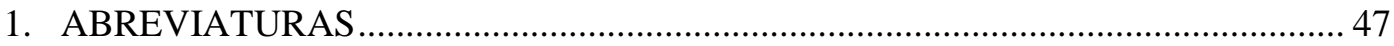

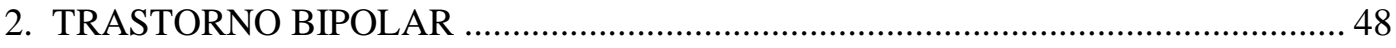

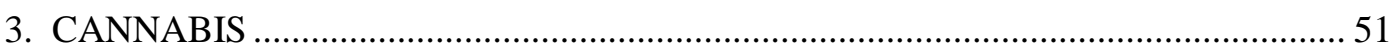




\section{RESUMEN}

En los pacientes con trastorno bipolar existe una elevada prevalencia de consumo de drogas, siendo el cannabis una de las principales. El consumo de cannabis puede modificar las manifestaciones clínicas y el curso evolutivo del trastorno. El objetivo del presente trabajo es estudiar la influencia del consumo de cannabis sobre la evolución del trastorno bipolar a partir de estudios de seguimiento de pacientes bipolares. Se ha realizado una revisión sistemática realizando una búsqueda de artículos en Medline. Se han obtenido 5 artículos sobre cohortes de sujetos bipolares que estudian el efecto de dicho consumo. El consumo de cannabis se presenta como un factor pronóstico negativo, con menor recuperación clínica, peor funcionamiento global, más tiempo en episodios afectivos y, posiblemente, mayor frecuencia de ciclos rápidos y episodios maníacos o mixtos. El cese del consumo durante el seguimiento mejora la evolución. El consumo de alcohol y otras drogas son muy frecuentes entre los consumidores de cannabis y se asocian a una evolución negativa.

Palabras clave: trastorno bipolar, consumo de cannabis, evolución.

\section{ABSTRACT}

Bipolar disorder patients have a high prevalence of drug use, being cannabis one of the main drugs involved. Cannabis use can modify clinical characteristics and outcome of this disorder. The aim of the current work is to evaluate from longitudinal studies the influence of cannabis use in the outcome of bipolar disorder. A systematic review was carried out through a Medline searching. 5 papers were found on bipolar patients cohorts that study this effect. Cannabis use is a negative prognostic factor, it was associated with less sucessful clinical recovery, worse global functioning, more time in affective episodes, and probably, more often rapid cycling and manic/mixed episodes. Bipolar patients who stop using cannabis show a better outcome. Alcohol and other drugs use are common between cannabis users and are associated with a negative outcome.

Key words: bipolar disorder, cannabis use, outcome. 


\section{INTRODUCCIÓN}

\subsection{TRASTORNO BIPOLAR}

El trastorno bipolar (TB) es un trastorno psiquiátrico grave, altamente incapacitante, que produce una elevada discapacidad, fundamentalmente entre adultos jóvenes, que se caracteriza por fluctuaciones en el estado de ánimo, con episodios recurrentes de depresión y estados de ánimo elevado (manía o hipomanía) (Grande et al. 2016). Se suele clasificar en TB tipo I (requiere de por lo menos un episodio maníaco), el TB tipo II (requiere una historia de episodios depresivos mayores e hipomaníacos) y TB sin especificar (incluyendo presentaciones que no encajan en las anteriores). El trastorno ciclotímico o ciclotimia (se incluye dentro de los TB en el DSM5, pero se considera aparte en la CIE-10, aunque dentro de los trastornos del humor) se caracteriza por fluctuaciones del estado de ánimo sin llegar a cumplir criterios de depresión mayor ni de hipomanía o manía (Criterios DSM 5 y CIE-10 en anexo 2, tablas 1,2 y 3). Existen otras formas como TB tipo III, ciclos rápidos, manía unipolar, etc. En el anexo 2, tabla 4 (de Dios et al. 2014) se especifican las diferencias entre DSM5 y el próximo CIE-11.

\subsubsection{Historia.}

Las primeras descripciones de estados de "intensa locura con exaltación del humor" nos llegan de los antiguos griegos. Ya en el siglo I a.C. se empiezan a relacionar estos estados con la melancolía, así, Sorano de Éfeso creía que la depresión y la manía eran dos entidades distintas pero que compartían síntomas prodrómicos y requerían tratamientos similares. Y, Areteo de Capadocia (siglo I d.C.), figura como el primero en señalar que la manía y la depresión formaban parte de un único trastorno. En el siglo XIX, la escuela de psiquiatría francesa realiza un gran avance en la concepción nosológica de estos trastornos. Jean Pierre Falret, en 1854, fue el primero en describir la "locura circular" (folie circulaire) como estados depresivos y maníacos separados por un intervalo libre, destacando el curso intermitente, el comienzo frecuentemente brusco y el carácter reversible de la mayoría de los episodios. Prácticamente al mismo tiempo y en el mismo lugar (La Salpetrière, París), Jules Baillarger (1854) describe la "locura de doble forma" (folie a double forme) en la misma línea que Falret. Emil 
Kraepelin, en 1896, introdujo el concepto de "locura maníaco-depresiva" como entidad nosológica independiente. Incluyó en un concepto único todas las psicosis que hasta entonces se habían denominado periódicas y circulares. Para él, todos estos cuadros son manifestaciones de la misma enfermedad. Al decir esto se basa en el carácter hereditario (historia familiar), la sintomatología afectiva similar, la sucesión periódica de fases maníacas o melancólicas con períodos de remisión y un pronóstico global comparativamente benigno sin un deterioro progresivo. Fue precisamente el pronóstico la principal característica que distinguía la locura maníaco-depresiva, delimitándola de la demencia precoz. Leonhard (1959) incluye la polaridad como factor decisivo en la diferenciación de las psicosis afectivas, distinguiendo aquellos pacientes depresivos que han tenido un episodio maníaco previo (bipolares) de aquellos que han sido siempre depresivos (unipolares) (Angst y Marneros, 2001; Bourgeois y Verdoux, 1997).

\subsubsection{Epidemiología.}

La prevalencia del TB tipo I se sitúa entre el 0,4 y el 1,6\% de la población general, siendo igual para ambos sexos y entre grupos étnicos. Los episodios de manía son más frecuentes en varones y los depresivos en mujeres. La prevalencia del TB tipo II estaría en torno al 0,5 y 1,9\% de la población, siendo más prevalente en mujeres. La edad de inicio del TB varía entre la infancia y los 50 años, con una edad media de 22 años, siendo los más frecuente entre finales de la adolescencia e inicios de la edad adulta (Uribe y Zorrilla, 2009).

\subsubsection{Etiopatogenia.}

En la etiopatogenia del TB influyen factores genéticos, factores sociales y ambientales (la adversidad social o situaciones estresantes precoces pueden favorecer el desarrollo del trastorno) y factores neurobiológicos.

Estudios de gemelos han encontrado que el TB es uno de los trastornos médicos con mayor carga genética. 


\subsubsection{Clínica.}

La característica clínica del TB se basa en la alternancia de episodios depresivos y maníacos/hipomaníacos a lo largo de la evolución de la enfermedad, pudiendo tratarse de episodios de distintos tipos: manía, hipomanía, depresión y estados mixtos.

Manía:

La manía se caracteriza por una exaltación del estado de ánimo. El humor suele ser eufórico, pero puede haber cambios rápidos del humor e inestabilidad afectiva o un humor disfórico (depresivo-irritable). El pensamiento es excesivamente positivo, mostrando una autoestima aumentada, con un sentido de grandiosidad y excesiva confianza en sí mismo. A nivel del lenguaje existe una verborrea o incontinencia verbal, con presión del habla (es difícil frenar su discurso). A nivel de la psicomotricidad, hay un aumento de la actividad, con aumento de la energía y disminución de la necesidad de sueño. Pueden aparecer ideas delirantes generalmente relacionadas con capacidades excepcionales como la riqueza y poderes sobrenaturales, son los denominados delirios congruentes con el estado de ánimo. También pueden aparecer delirios de referencia o persecución, denominados delirios incongruentes con el estado de ánimo. Pueden presentarse fenómenos alucinatorios, generalmente auditivos y, en situaciones de manía grave, puede aparecer un discurso incomprensible.

\section{Hipomanía:}

Es una forma atenuada del estado maníaco, donde existe una elevación del humor menos intensa, con un incremento en los niveles habituales de energía y actividad, pero que, a diferencia de la manía, no requiere ingreso hospitalario.

\section{Depresión:}

El estado depresivo en el TB tiene características similares a la depresión mayor unipolar. En general, las características clínicas de la depresión se pueden dividir en:

1. Estado de ánimo: triste, melancólico, preocupado, irritable.

2. Estado cognitivo: pérdida de interés, dificultades en la concentración, baja autoestima, pensamientos negativos, indecisión, culpa, ideas suicidas.

3. Conducta: inhibición psicomotriz, conducta suicida. 
4. Somático (físico): trastornos del sueño, fatiga, disminución del apetito, pérdida de peso, dolor, molestias gastrointestinales, disminución de la libido.

\section{Episodios mixtos:}

Se caracterizan por la aparición simultánea de sintomatología maníaca y depresiva en el mismo episodio. Es un cuadro caracterizado por inquietud psicomotriz, insomnio, taquipsiquia y verborrea, combinada con pensamiento depresivo, llanto y labilidad emocional.

Algunos autores señalan que dentro de la manía se incluye la dimensión depresiva que puede aparecer incluso en los cuadros de manía pura, que se caracteriza por humor deprimido, ideas de suicidio, culpa, síntomas obsesivos y ansiedad psíquica (González-Pinto et al. 2003).

\subsubsection{Evolución.}

Es un trastorno crónico recidivante, generalmente con remisión clínica interepisódica, pero muchos pacientes presentan síntomas subsindrómicos entre los episodios. Es una enfermedad que produce una elevada discapacidad sociolaboral. Un elevado porcentaje, sobre $10-20 \%$ de los pacientes, puede fallecer por suicidio, habitualmente en fases depresivas o mixtas. Otro porcentaje puede evolucionar hacia un deterioro similar a la esquizofrenia.

\subsubsection{Diagnóstico.}

El diagnóstico es a través de la entrevista clínica, siendo fundamental la valoración longitudinal del cuadro para determinar el tipo de episodios que ha sufrido.

El diagnóstico diferencial se realiza con otros trastornos mentales como la depresión unipolar, la esquizofrenia, el trastorno esquizoafectivo o el trastorno límite de personalidad. Hay que descartar causas de manía secundaria debido a enfermedades médicas (neurológicas, endocrinas), inducidas por drogas (psicoestimulantes) o por otros fármacos (corticoides, antidepresivos, antihipertensivos). 


\subsubsection{Tratamiento.}

El tratamiento de elección es el litio. También están indicados los anticonvulsivantes como el ácido valproico o la lamotrigina y algunos antipsicóticos como la olanzapina. En la práctica clínica es necesaria con frecuencia la combinación de distintos fármacos. Los episodios mixtos parecen responder mejor al ácido valproico. Los antidepresivos en la depresión bipolar se han relacionado con el fenómeno de viraje a manía. Cuando se da la existencia de un episodio grave resistente al tratamiento o con aparición de síntomas psicóticos existe indicación de terapia electroconvulsiva.

Un problema habitual es la falta de adherencia a la medicación, lo que empeora la evolución. La falta de adherencia se ha relacionado con la presencia de síntomas psicóticos, pobre insight, problemas laborales y consumo de cannabis (González-Pinto et al. 2010).

\subsection{CANNABIS}

El cannabis es una droga que se consume fundamentalmente fumada como marihuana (hace referencia a los hojas y flores de la planta desecadas) o como hachís (procede de la resina secretada por la planta). Es la droga ilegal más usada en todos los países de nuestro entorno. España es uno de los países del mundo de mayor consumo de cannabis. En el cannabis se han identificado más de 400 sustancias diferentes, de las cuáles hasta 100 pertenecen químicamente al grupo de los cannabinoides. El $\Delta 9$ Tetrahidrocannabinol (THC) es el principal componente psicoactivo de la planta. El cannabis es menos dañino para la salud física que otras drogas, pero es una de las más nocivas respecto a posibles consecuencias psiquiátricas (Bobes y Calafat 2000).

\subsubsection{Sistema endocannabinoide.}

El cannabis actúa sobre el recientemente descrito sistema cannabinoide, ampliamente extendido en el cerebro. En la actualidad están identificados dos receptores cannabinoides: el CB1 y el CB2. En el cerebro, el receptor CB1 se localiza principalmente en cerebelo y ganglios basales indicando un papel en la coordinación motora, en hipocampo con un papel en la memoria, en el córtex en relación con la regulación de funciones cognitivas, en tálamo regulando mecanismos del dolor y las 
emociones o en hipotálamo regulando el apetito. Los dos ligandos endógenos principales son la anandamida y el 2-araquidonil-glicerol (Ramos et al. 2017).

\subsubsection{Epidemiología.}

La dependencia de cannabis a lo largo de la vida se estima en un 1.5-2.5\%. El consumo alguna vez en la vida entre jóvenes es del 35\%, en el último mes de 9\% y diariamente del $2 \%$. La edad de inicio en el consumo se calcula que es a los 15 años como promedio según la última encuesta española en estudiantes de educación secundaria (Anexo 2 figuras 1,2$)$.

\subsubsection{Características clínicas del consumo de cannabis.}

Intoxicación: comienza pocos minutos después de haberlo fumado y dura 1-2 horas. Produce generalmente un efecto bifásico: una fase inicial de estimulación (euforia, bienestar, aumento de la percepción), y una segunda fase donde predomina la sedación (relajación, somnolencia). Se produce una agudización de las percepciones visuales, auditivas y táctiles, así como una ligera distorsión del espacio y tiempo. También aparece risa fácil, locuacidad y aumento del apetito, con preferencia por los dulces. En la esfera cognitiva, disminuye la capacidad de concentración, altera la memoria reciente y la resolución de tareas complicadas. Como signos físicos se presenta el enrojecimiento conjuntival, la boca seca y la taquicardia. En algunos sujetos, especialmente en consumidores inexpertos o tras dosis elevadas, puede aparecer síntomas de ansiedad, disforia o ideación paranoide (Anexo 3, tablas 5 y 6).

Abstinencia: El cuadro se describe en más del 50\% de los consumidores intensos o en el 15\% de los consumidores regulares y, en algunas ocasiones, con una clínica muy florida. Aparece irritabilidad, nerviosismo, insomnio, pesadillas, pérdida de apetito, sudoración, temblor y un intenso deseo de consumo de cannabis (Anexo 3, tabla 7).

Dependencia: La dependencia a una droga se caracteriza por la presencia de un deseo intenso de consumo, una pérdida de control sobre dicho consumo y un repertorio conductual destinado a la obtención y el consumo de la droga (Anexo 3, tablas 8, 9 y 10). Aunque los criterios diagnósticos son iguales para todas las drogas puede haber diferencias entre ellas, así la tolerancia es menos frecuente en el caso de los efectos 
subjetivos del cannabis. Sin embargo, existe tolerancia para la mayoría de los efectos físicos del cannabis, como son la taquicardia, el descenso de temperatura de la piel o el descenso de la presión intraocular.

\subsubsection{Tratamiento de la dependencia de cannabis.}

El tratamiento de deshabituación se refiere a la fase de tratamiento que se realiza después de la desintoxicación y que está dirigida a mantener la abstinencia en la sustancia y evitar la recaída en el consumo. Los tratamientos usados son psicoterapia y psicofármacos. Son frecuentes las recaídas, de forma similar a otras adicciones (Roffman y Stephens, 2006).

\subsection{CONSUMO DE DROGAS Y TRASTORNO BIPOLAR}

La patología dual hace referencia a la coexistencia de un trastorno mental y una adicción (Goikolea y Vieta, 2012). El TB es la enfermedad mental que con mayor frecuencia se asocia a la comorbilidad con TUS (Regier et al., 1990). Cuando el TB se asocia con un TUS, el pronóstico empeora.

El estudio ECA (Epidemiologic Catchment Area) observó una prevalencia a lo largo de la vida del $56,1 \%$ para cualquier TUS en el total de la muestra de sujetos con TB. En los sujetos con TB tipo I, la prevalencia de TUS fue del 60,7\%, y en los de tipo II del 48,1\% (Regier et al., 1990). En otro estudio epidemiológico norteamericano, el National Comorbidity Survey (NCS), el riesgo de dependencia de drogas era 8 veces más elevado en los sujetos bipolares respecto al resto de la población general (Kessler et al., 1997), sobre todo en el TB tipo I. En otro estudio epidemiológico, el National Epidemiologic Survey on Alcohol and Related Conditions (NESARC), las cifras de prevalencia son muy elevadas, así la prevalencia de trastornos por uso de alcohol entre los sujetos con TB fue del 58\% a lo largo de la vida 23,6\% el año previo, y la de trastornos por uso de otras sustancias del $37.5 \%$ (Grant et al., 2005).

Los sujetos con TB tienden a consumir más sustancias de todo tipo en las fases maníacas. Las drogas que más consumen son el alcohol y el cannabis, seguido de la cocaína (Cerullo y Strakowski, 2007). 


\subsubsection{Etiopatogenia.}

Se han propuesto diversas hipótesis para explicar las elevadas cifras de comorbilidad entre el TB y el TUS (Goikolea y Vieta, 2012): El consumo de sustancias psicoactivas puede desencadenar episodios de manía o depresión; los síntomas de hipomanía o manía incluyen la implicación excesiva en actividades placenteras que incluyen el consumo de sustancias; la vulnerabilidad genética común para ambos trastornos, factores neurobiológicos comunes o la vulnerabilidad social común (situaciones vitales adversas); la hipótesis de la automedicación (los pacientes bipolares consumirían sustancias como medio para aliviar síntomas de la enfermedad) y los rasgos de personalidad comunes para ambos trastornos.

\subsubsection{Efecto del trastorno por uso de sustancias comórbido sobre la clínica y el curso del trastorno bipolar.}

Los pacientes con TB y consumo de sustancias suelen presentar una serie de complicaciones en comparación con el TB sin comorbilidad con TUS, que lo convierte en más grave y difícil de tratar (Goikolea y Vieta, 2012).

Los pacientes con TB que consumen sustancias tienen una mayor probabilidad de sufrir episodios mixtos (Goldberg et al., 1999) y más probabilidades de requerir hospitalizaciones a lo largo del curso evolutivo de la enfermedad (Cassidy et al., 2001). También se ha descrito que los pacientes bipolares duales sufren más episodios afectivos y más ciclación rápida (Prisciandaro et al., 2019). La comorbilidad con el abuso de sustancias es una de las principales variables que se ha relacionado con una mayor gravedad de los episodios maníacos. Por otro lado, distintos estudios han relacionado la suicidalidad con la comorbilidad con TUS en el TB (González-Pinto et al., 2006), tanto en el caso del TB tipo I como en el tipo II. Los pacientes bipolares duales tienen un peor funcionamiento global y un estatus ocupacional más bajo (Prisciandaro et al. 2019), además de peor adherencia al tratamiento (González-Pinto et al., 2006).

La presencia de un TUS empeora el curso del TB y conlleva una peor respuesta a los tratamientos eutimizantes. Además, los pacientes bipolares que padecen un TUS presentan peor evolución del consumo (Goikolea y Vieta, 2012). 


\subsection{CANNABIS Y TRASTORNO BIPOLAR}

Como hemos señalado el TB es uno de los trastornos más asociados con el consumo de drogas y, de estas, el cannabis es una de las drogas más frecuentes y dicho consumo puede tener relevancia en las manifestaciones clínicas y en la evolución del TB.

\subsubsection{Epidemiología.}

En el estudio NESARC se obtuvo una prevalencia de trastorno por uso de cannabis (TUC) en el último año de $2.5 \%$ y de $6.3 \%$ a lo largo de la vida. El riesgo de tener un trastorno mental entre los consumidores de cannabis era de una OR de 2.5 y entre aquellos con TUC de 3.2. Las relaciones fueron principalmente con otros TUS, algunos trastornos de personalidad y el TB. Los sujetos con un trastorno mental en el último año constituían el $72 \%$ de los consumidores de cannabis y consumían el $83 \%$ del cannabis (Lev-Ran et al, 2013b).

El TB es uno de los cuadros más relacionados con el TUC. Por un lado, existe una elevada prevalencia de consumo y dependencia en pacientes bipolares. En una revisión se señala una prevalencia del 30-64\% de consumo de cannabis a lo largo de la vida entre pacientes bipolares (Ashton et al. 2005). En el NESARC, entre sujetos con TB había un 7.2\% de TUC en los últimos 12 meses (Lev-Ran et al. 2013a). Otros autores estiman un 70\% de consumo de cannabis a lo largo de la vida y un 30\% de TUC en el TB (Bally et al. 2014).

\subsubsection{Dependencia de cannabis y trastorno bipolar.}

Se ha descrito un mayor riesgo de episodios maníacos y TB en adictos (Arendt et al., 2007, Henquet 2006). Se ha relacionado el mayor riesgo de aparición de un TB con la dosis de cannabis y la edad de inicio en el consumo más precoz. Igualmente, el consumo de cannabis adelanta la edad de inicio del TB (Lagerberg et al. 2011), apoyando al menos un papel precipitante. Además, se ha descrito la inducción de cuadros maníacos con los cannabinoides sintéticos (Ustundag et al. 2015).

Algunos autores han valorado las relaciones entre distintos estados de ánimo y consumo, así entre 24 bipolares en una evaluación de 6 días, observan como el ánimo expansivo lleva al consumo y éste lleva a la manía y la depresión (Tyler et al. 2015). 
Además, pueden existir distintos cambios cognitivos debidos al consumo de cannabis en pacientes con TB, como alteración en la toma de decisiones, en la memoria de trabajo o el tiempo de reacción como se sugiere en una revisión (Cahill et al. 2006). Sin embargo, otros estudios observan mejor situación cognitiva, aunque sí evidenciaban una peor evolución (Braga et al. 2012). Igualmente, otros autores señalan una mejor situación cognitiva, aunque confirman los hallazgos de un inicio más precoz de la manía con el consumo y peor evolución, con más episodios afectivos y más ciclos rápidos (Bally et al 2014).

Existen pocos estudios de seguimiento que valoren los efectos del consumo de cannabis en la evolución del TB y las repercusiones de la persistencia o el cese del consumo.

\section{HIPÓTESIS}

El consumo de cannabis empeora el pronóstico del trastorno bipolar.

El cese de dicho consumo mejora la evolución del trastorno bipolar.

Con mayor gravedad del consumo de cannabis, peor evolución del trastorno bipolar.

El consumo de cannabis se asocia con diferencias en las manifestaciones clínicas del trastorno bipolar durante el seguimiento.

\section{OBJETIVOS}

Valorar la repercusión del consumo de cannabis en la evolución del trastorno bipolar valorado por recaídas, ingresos, gravedad clínica, funcionalidad, adherencia al tratamiento o cambios en las características clínicas (ciclos rápidos, predominio de fases maniacas o depresivas, número de fases).

\subsection{OBJETIVOS SECUNDARIOS:}

- Evaluar la repercusión del consumo de cannabis sobre la evolución del trastorno bipolar según las características sociodemográficas: edad basal en la valoración y sexo del paciente. 
- Evaluar la repercusión según las características del trastorno bipolar: tipo de trastorno bipolar, gravedad del trastorno, edad de inicio del trastorno bipolar, predominio maniaco o depresivo.

- Evaluar la repercusión de distintas variables relacionadas con el consumo de cannabis: edad de inicio, potencia, dosis consumida, gravedad del consumo (uso, abuso, dependencia).

- Evaluar la repercusión de la presencia de policonsumo en los efectos del consumo de cannabis sobre el trastorno bipolar.

- Evaluar los efectos del cese del consumo de cannabis en la evolución del trastorno bipolar.

- Evaluar los efectos cognitivos del consumo de cannabis durante el seguimiento del trastorno bipolar.

- Evaluar la repercusión del consumo en la presencia de ciclos rápidos y manifestaciones clínicas atípicas.

- Evaluar la repercusión del consumo en la resistencia al tratamiento.

\section{METODOLOGÍA}

Se realizó una revisión sistemática sobre los efectos del consumo de cannabis en la evolución del trastorno bipolar. Se llevó a cabo la búsqueda a través de PubMed. PubMed es un motor de búsqueda para la base de datos MEDLINE de citaciones de artículos de investigación biomédica, ofrecido por la Biblioteca Nacional de Medicina de los Estados Unidos. MEDLINE tiene alrededor de 4800 revistas publicadas en más de 70 países desde 1966 hasta la actualidad.

Se usaron las siguientes estrategias de búsqueda en noviembre de 2018:

Bipolar disorder AND cannabis AND outcome: se obtuvieron 39 artículos.

Bipolar disorder AND cannabis AND relapse: se obtuvieron 15 artículos.

Bipolar disorder AND cannabis AND course: se obtuvieron 40 artículos. 


\subsection{CRITERIOS DE SELECCIÓN DE ARTÍCULOS}

- Inglés o español.

- Cualquier fecha.

- Cohorte de pacientes con trastorno bipolar y consumo de cannabis, que evalúen como variables evolutivas: recaídas del trastorno bipolar, ingresos, gravedad clínica durante el seguimiento, adherencia al tratamiento o cumplimiento con tratamiento o funcionalidad.

Para complementar la selección, se comprobaron las referencias de los artículos encontrados con la finalidad de explorar otros artículos de interés para el estudio. Esta estrategia no ofreció artículos de interés adicionales.

Tras suprimir los artículos duplicados se obtuvieron 76 artículos, que incluían:

- Trastornos mentales y trastorno por uso de sustancias en general (21)

- Tratamientos psiquiátricos (6)

- Trastorno mental no bipolar (8)

- Trastorno bipolar sin relación con trastorno por uso de sustancias (17)

- Trastorno por uso de sustancias en general (2)

- Cannabis sin relación con trastorno mental (1)

- Trastorno bipolar y trastorno por uso de sustancias en general (5)

- Trastorno bipolar y uso de cannabis sin seguimiento (7)

- Artículo de revisión sobre los efectos del cannabis en la evolución del trastorno bipolar (1)

- Seguimiento de un caso clínico de trastorno bipolar y consumo de cannabis (1)

- Artículo en holandés de una cohorte ya seleccionada (Van Rossum et al. 2009) (1)

- Subanálisis de datos de una cohorte ya seleccionada (Kvitland et al. 2016) (1)

Entre los artículos excluidos figuran dos artículos en alemán, uno en italiano, uno en francés y uno en portugués no relacionados con la temática de la revisión y un artículo en holandés ya mencionado.

Artículos incluidos (Kim et al. 2015, Kvitland et al. 2015, Strakowski et al. 2007, Van Rossum et al. 2009, Zorrilla et al. 2015) (5) 

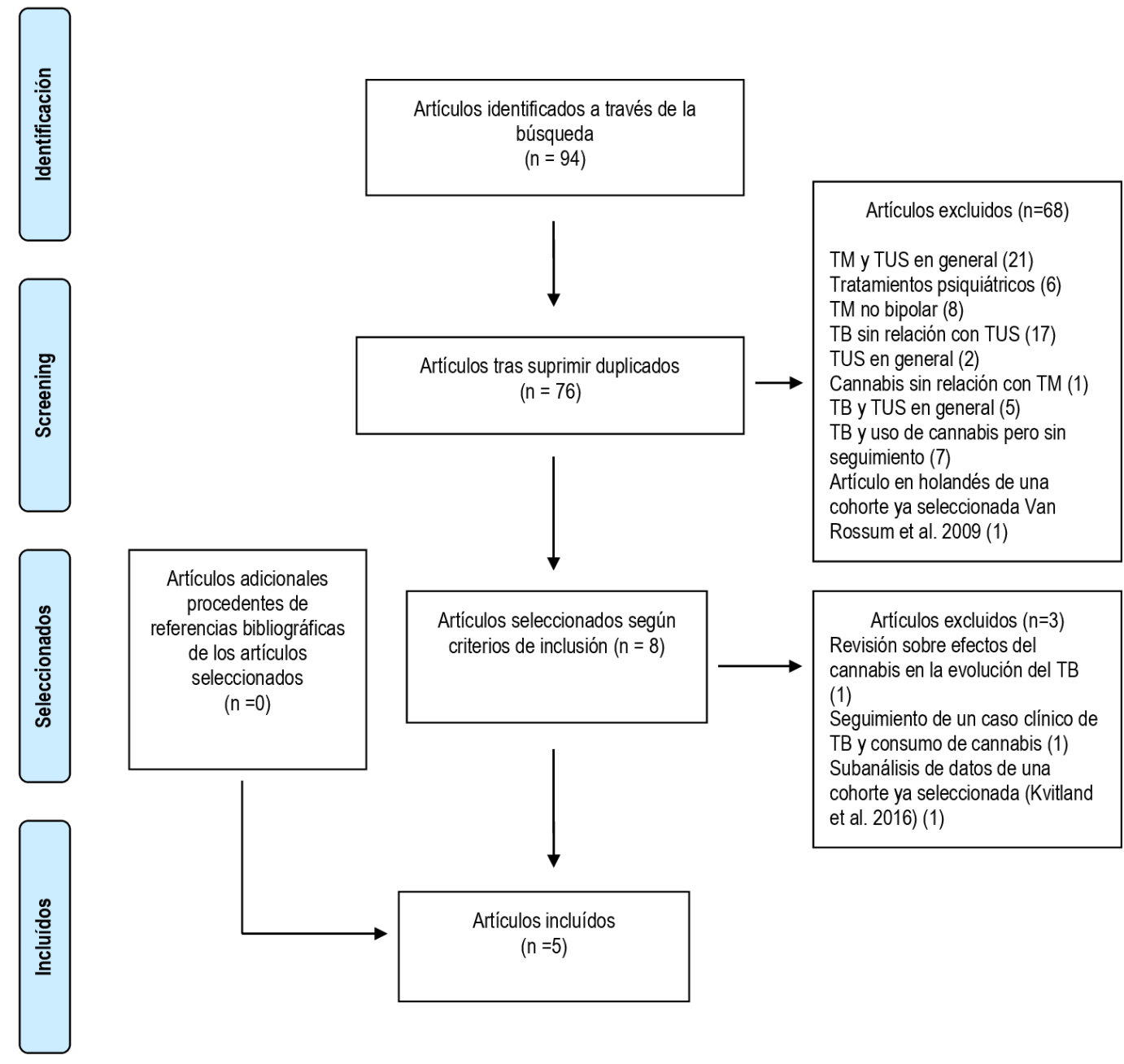

TB: Trastorno bipolar; TM: Trastorno mental; TUS: Trastorno por uso de sustancias

FIGURA 3. Diagrama PRISMA. 


\section{RESULTADOS}

\subsection{REPERCUSIÓN DEL CONSUMO DE CANNABIS EN LA EVOLUCIÓN DEL TRASTORNO BIPOLAR}

Se trata de valorar la repercusión del consumo de cannabis en la evolución del TB valorado por recaídas, ingresos, gravedad clínica, funcionalidad, adherencia al tratamiento o cambios en las características clínicas (predominio de fases maniacas o depresivas, número de fases).

El consumo de cannabis se asocia con una serie de repercusiones negativas en la evolución del TB (Tabla 11 y 12).

El consumo se asocia con una menor remisión clínica medida por las escalas de HAMD e YMRS durante los 24 meses de seguimiento (Kim et al. 2015). Por otro lado, el consumo continuado se asocia con peor funcionalidad (valorado por la escala GAF, así no alcanzan la recuperación funcional el $67 \%$ en el grupo de consumo continuado frente al $29 \%$ en el grupo de no consumidores, pero no es estadísticamente significativo en el análisis bivariante, pero si al controlar por otros factores de confusión) y se asocia con más intensidad de la manía (mayor puntuación en la escala YMRS) a los 12 meses. Las diferencias en algunas medidas no son significativas por el escaso número de consumidores durante el seguimiento (Kvitland et al. 2015).

En otra cohorte, el consumo se asocia con una mayor duración de los episodios afectivos y más ciclos rápidos, aunque no había relación con el porcentaje de recuperación y recurrencia a los 60 meses de seguimiento (Strakowski et al. 2007). Mientras que, otro estudio, observa que el consumo se asocia con una menor recuperación, menor remisión, mayor recurrencia y peor funcionamiento laboral a los 24 meses de seguimiento, además, los autores observan que si cesa el consumo la evolución mejora. Así, los consumidores previos tenían mayores porcentajes de remisión (68\%), recuperación (38.7\%) y menor porcentaje de recurrencia (42\%) y recaída (29.8\%), siendo una evolución similar al grupo de nunca consumidores. Los consumidores activos tenían menor recuperación y remisión, mayor recurrencia y mayor deterioro laboral y de no vivir con pareja en el modelo de regresión ajustado por características basales, aunque las diferencias se atenúan cuando se ajusta por otros 
consumos de sustancias (Zorrilla et al. 2015). Por último, otro estudio procedente de la misma cohorte que el anterior, observa que los consumidores de cannabis tenían mayor gravedad de la enfermedad global durante el seguimiento medida por la CGIBP ajustada por otras variables como la gravedad basal, además de mayor gravedad de la manía y de los síntomas psicóticos, pero no de la depresión y menos satisfacción con la vida a los 12 meses de seguimiento (Van Rossum et al. 2009).

Respecto al cumplimiento del tratamiento durante el seguimiento de los pacientes bipolares, algunos valoran el cumplimiento, pero no presentan datos (Zorrilla et al. 2015), otros no observan diferencias en el cumplimiento (Strakowski et al. 2007), ni diferencias en completar el seguimiento al año (Strakowski et al. 2007). Mientras que un estudio, observa un menor cumplimiento con el tratamiento en el grupo de consumidores (Van Rossum et al. 2009).

\subsection{CARACTERÍSTICAS BASALES DIFERENCIALES ENTRE LOS CONSUMIDORES Y NO DE CANNABIS}

Se trata de analizar las diferencias de la clínica, la gravedad o la edad de inicio del TB entre los consumidores y no de cannabis, previo al inicio del seguimiento.

El trabajo de Kim et al. (2015) encuentra que los consumidores tenían mayor gravedad inicial, mayor puntuación en la escala de manía y depresión, aunque no había diferencias en la edad de inicio del TB, ni diferencias en características clínicas como la ideación suicida o la presencia de síntomas psicóticos.

Strakowski et al. (2007) diferencian dos subgrupos entre los pacientes bipolares consumidores de cannabis, aquellos donde el TB se manifiesta primero (bipolar primero) de los que presentan el consumo antes que el TB (cannabis primero), siendo la edad de inicio del TB distinto en los dos grupos (más tardía en el subgrupo de cannabis primero). En los consumidores había más presencia de síntomas psicóticos, pero no había diferencias en la puntuación de las escalas de depresión o manía (HAMD, YMRS) ni diferencias en la presencia de episodios mixtos previos.

Van Rossum et al. (2009) observan un inicio más precoz del TB en los consumidores (24 años frente a 31 años), mayor gravedad inicial en la valoración de manía y síntomas psicóticos y había un mayor porcentaje de ingresados frente a pacientes ambulatorios. 
Zorrilla et al. (2015) con un estudio basado en la misma cohorte que el de Van Rossum et al. (2009), observan que los consumidores de cannabis activos tienen mayor número de episodios maniacos previos, mayor insatisfacción con la vida, mayor gravedad global y de la manía, más presencia de cicladores rápidos y mayor frecuencia de intentos de suicidio previos. Ambos grupos de consumidores tenían mayor frecuencia de pacientes ingresados, menor proporción de sujetos viviendo con pareja, más síntomas psicóticos, más intentos de suicidio, una edad de inicio del TB más precoz, más ingresos previos y peor adherencia a la medicación. No había diferencias en la presencia de síntomas depresivos.

Kvitland et al. (2015) no encuentran diferencias en la presencia de síntomas depresivos o psicóticos durante el seguimiento y refieren que no hay diferencias basales en los síntomas, aunque no ofrecen datos sobre estos.

\subsection{INFLUENCIA DE LAS CARACTERÍSTICAS SOCIODEMOGRÁFICAS EN EL EFECTO DEL CONSUMO SOBRE LA EVOLUCIÓN DEL TRASTORNO BIPOLAR}

Se trata de valorar si el efecto del consumo de cannabis en la evolución del TB está influido por la edad en el momento de la valoración y el sexo de los participantes.

En todos los trabajos los consumidores son más jóvenes y presentan un predominio de varones respecto a los no consumidores, por lo que al haber pocas mujeres consumidoras no suele realizarse este análisis.

Los trabajos evaluados no valoran la edad basal como posible predictor de la evolución. Strakowski et al. (2007) diferencian el subgrupo de cannabis primero de bipolar primero, de los cuáles los primeros son más mayores en la evaluación basal pero no analizan el efecto sobre la evolución, aunque hay algunas diferencias en la evolución entre el subgrupo de bipolar primero y cannabis primero. Así los consumidores de cannabis, más los del subgrupo de bipolar primero, están más tiempo en episodios afectivos (maniacos y mixtos), mientras que los del subgrupo de cannabis primero presentan mejor recuperación, pero no tras ajuste por otras variables (por sexo, edad de inicio del TB). Las diferencias lo atribuyen a la distinta secuencia en el inicio de los trastornos, más que a las diferencias en la edad de los subgrupos. 
Tampoco suele haber análisis por sexo. En el trabajo de Kvitland et al. (2015) sólo hay una mujer en el grupo de consumidores. El único que lo analiza es el trabajo de Kim et al. (2015) que señala que las mujeres consumidoras tienen menor remisión total, pero sólo hay 9 mujeres para el análisis. Observan un efecto diferencial, con menor remisión en la escala de HAMD en las consumidoras mujeres y menor remisión en la escala de YMRS en los consumidores varones respecto a los no consumidores.

\subsection{INFLUENCIA DE LAS CARACTERÍSTICAS DEL TRASTORNO BIPOLAR EN LOS EFECTOS DEL CONSUMO DE CANNABIS EN LA EVOLUCIÓN}

Se trata de valorar si las características del TB influyen en los efectos del consumo de cannabis sobre la evolución del primero.

La mayoría de los trabajos valoran pacientes con TB tipo I, generalmente en fase maníaca. Kim et al. (2015) incluye también pacientes con trastorno esquizoafectivo tipo bipolar y no observan diferencias evolutivas respecto a los bipolares.

En general, no valoran la influencia de la gravedad inicial de los cuadros en la evolución posterior. No se valora la repercusión del tipo de fases predominantes previas (maniacas, depresivas, mixtas).

Tampoco valoran la repercusión de la edad de inicio del TB como posible factor pronóstico en las repercusiones del consumo de cannabis sobre el TB.

\subsection{INFLUENCIA DE LAS CARACTERÍSTICAS DEL CONSUMO DE CANNABIS EN LOS EFECTOS DEL CANNABIS EN LA EVOLUCIÓN}

Se trata de valorar si las características del consumo de cannabis repercuten en la evolución del TB, datos sobre la gravedad del consumo, la potencia del cannabis, la edad de inicio en el consumo o el tiempo de consumo.

Los criterios de consumo de cannabis son dispares entre los distintos estudios y no comparables. La definición de consumo en el trabajo de Kim et al. (2015) es de consumo 3 o más días por semana, mientras que Strakowski et al. (2015) si utilizan los criterios establecidos de abuso o dependencia mediante la entrevista SCID. 
La prevalencia de consumidores en las muestras son variables, un $10.7 \%$ de consumidores en la muestra de Kim et al. (2015) (consumidores de 3 días o más por semana, aunque sólo 3 de los 25 consumidores no fumaban diariamente) y hasta un 47.9\% de abuso-dependencia en la muestra de Strakowski et al. (2015).

En el trabajo de Kvitland et al. (2015), de los 62 pacientes incluidos, 7 consumían al principio, 2 durante el seguimiento y sólo 6 consumían al principio y al final por medio de una entrevista. Valoran cualquier consumo de cannabis durante el seguimiento, aunque la media es de 2-3 veces por semana. Refieren que el consumo a lo largo de la vida de cannabis en la muestra es del $52 \%$.

Zorrilla et al. (2015) establecen los criterios de uso, abuso y dependencia, pero para el análisis estadístico lo agrupan y no diferencian los posibles efectos por separado. En su muestra existe un $6.9 \%$ de consumidores de cannabis activos, $4.6 \%$ consumidores previos y $88.5 \%$ de nunca consumidores

Van Rossum et al. (2009) valoran uso, abuso y dependencia por autoinformes, pero reagrupan también en dos grupos. También valoran el consumo durante el seguimiento y suman la gravedad, pero no ofrecen datos sobre las repercusiones en la evolución o sobre las repercusiones de mantener el consumo. En la visita basal hay un 54.5\% de consumidores de cannabis, parece que hacen referencia a cualquier uso de la sustancia, $27.5 \%$ con criterios de abuso y $6.8 \%$ con criterios de dependencia de cannabis. Al final del seguimiento había un descenso a $27.5 \%$ de consumo de cannabis, $10.1 \%$ de abuso de cannabis y $0.6 \%$ de dependencia de cannabis.

La mayoría de los autores no presentan datos sobre cantidad de consumo, frecuencia de consumo o la potencia (es decir, el contenido de THC) del cannabis utilizado y no se analiza la posible influencia en la evolución.

La edad de inicio en el consumo no se valora como factor pronóstico en ninguno de los estudios analizados. Strakowski et al. (2007) diferencian el subgrupo de cannabis primero con edad de inicio a los 16 años y los bipolares primero con edad de inicio a los 18 años, aunque la diferencia no es significativa y no valoran el efecto de la edad de inicio en la evolución. 


\subsection{EFECTOS DEL POLICONSUMO EN LOS EFECTOS DEL CONSUMO DE CANNABIS SOBRE EL TRASTORNO BIPOLAR}

Se trata de valorar la influencia del consumo concomitante de otras drogas distintas al cannabis en la evolución del TB.

Kvitland et al. (2015) no ofrecen datos sobre el policonsumo, aunque si se valoran en una publicación posterior del mismo estudio, pero no sus efectos sobre la evolución (Kvitland et al. 2016).

Kim et al. (2015) ofrecen datos sobre el consumo de alcohol y tabaco. No hay diferencias significativas en el porcentaje de abuso o dependencia de alcohol entre consumidores y no de cannabis. Tampoco observan diferencias en la evolución del TB debido al consumo de alcohol, pero sólo hay 8 sujetos con abuso o dependencia de alcohol entre los 25 consumidores de cannabis. Encuentran que la presencia concomitante de consumo de tabaco y cannabis se asocia con una menor remisión.

Zorrilla et al. (2015) encuentran que los consumidores de alcohol se distribuyen en un $37.7 \%$ entre los consumidores de cannabis actual, un $13.4 \%$ en consumidores de cannabis previo y un $49 \%$ entre los no consumidores de cannabis. Otras drogas se distribuyen en un $3.7 \%$ entre los consumidores de cannabis actual, un $4.4 \%$ entre los consumidores de cannabis previo y un $91.9 \%$ entre los no consumidores. No ofrecen datos sobre tipos de drogas. El consumo de alcohol y otras drogas tiene efectos negativos en la evolución funcional y se asocian con menor recuperación y remisión. En el análisis multivariante ajustado por el efecto de estas drogas, el efecto de cannabis en la evolución se atenúa, sobre todo en la evolución funcional. El consumo actual de cannabis se asocia sólo con una mayor recurrencia en este modelo multivariante donde se incluyen el consumo de otras drogas.

Van Rossum et al. (2009), con datos de la misma cohorte que el estudio de Zorrilla et al. (2015), valoran igualmente consumo de alcohol y otras drogas en la valoración basal y en el seguimiento. Diferencian uso, abuso y dependencia, pero no tienen en cuenta la diferencia de gravedad para el análisis de los resultados. Los consumidores de cannabis presentan un mayor consumo de alcohol y de otras drogas. Hay un $80.3 \%$ de consumo de alcohol basal en los consumidores de cannabis y un 37.2\% entre los no consumidores y un $34.3 \%$ de consumo de otras drogas entre los consumidores de 
cannabis y un $2.6 \%$ entre los no consumidores, ambos disminuyen durante el seguimiento. El consumo de otras drogas modifica los efectos del consumo de cannabis sobre el cumplimiento del tratamiento y la satisfacción con la vida.

Strakowski et al. (2007) indican que un 60\% del subgrupo de bipolar primero tienen abuso o dependencia de alcohol y un 70\% del subgrupo de cannabis primero, además presenta más gravedad del consumo de alcohol en este último. Pero, incluso en el subgrupo de no consumidores de cannabis, un $22 \%$ tenían abuso o dependencia de alcohol. Al ajustar el análisis estadístico por el consumo de alcohol, el consumo de cannabis se asocia con la duración de la depresión, con menos tiempo en remisión afectiva y presencia de síntomas subsindrómicos. Respecto a otras drogas, no había entre el grupo de no consumidores de cannabis y había 11 en los otros dos subgrupos, en un $8 \%$ de los bipolares primeros y en un $24 \%$ del subgrupo de cannabis primero, pero no valoran la asociación con la evolución del TB.

\subsection{EFECTOS DEL CESE DEL CONSUMO EN LA EVOLUCIÓN DEL TRASTORNO BIPOLAR}

Se trata de valorar los efectos sobre la evolución del TB de la persistencia o el cese del consumo durante el seguimiento.

La persistencia en el consumo durante el seguimiento no lo valora el estudio de Kim et al. (2015), otros autores valoran la persistencia en el consumo, pero no analizan el efecto (Van Rossum et al. 2009).

Strakowski et al. (2007) observan que un 70\% de los consumidores se recuperan del consumo tras el ingreso por manía (es decir, al menos 8 semanas sin consumo o poco intenso) pero se produce un $60 \%$ de recurrencia en el consumo posterior, lo que sugieren que es una época adecuada para la intervención terapéutica. Además, señalan la aparición de 16 nuevos casos de trastorno por uso de cannabis durante el seguimiento, pero consideran que se parecen al resto de grupo de no consumidores.

En otro estudio, el objetivo principal del trabajo es valorar el efecto de la persistencia en el consumo de cannabis sobre la evolución. El mantenimiento del consumo se asocia con una mayor puntuación en la escala de manía (YMRS) y peor 
funcionamiento medido por la escala GAF, aunque no estadísticamente significativo (Kvitland et al. 2015),

El objetivo principal del trabajo de Zorrilla et al. (2015) es también valorar el efecto del cese del consumo de cannabis en la evolución del TB. Señalan que los consumidores de cannabis previo tenían mayores porcentajes de remisión, recuperación y menor porcentaje de recurrencia y recaída que los consumidores activos durante el seguimiento, con una evolución similar a los nunca consumidores. Los consumidores activos tenían menor recuperación y remisión, mayor recurrencia y mayor deterioro laboral y no vivir con pareja en el modelo de regresión logística, aunque estos efectos eran menos manifiestos cuando se ajustaba por el efecto de otras drogas.

\subsection{EFECTOS COGNITIVOS DEL CONSUMO DE CANNABIS DURANTE EL SEGUIMIENTO DEL TRASTORNO BIPOLAR}

Se trata de valorar si el consumo de cannabis repercute en la evolución del deterioro neuropsicológico en los sujetos con TB. No lo valora ninguno de los estudios revisados.

\subsection{EFECTOS DEL CONSUMO DE CANNABIS EN LA PRESENCIA DE CICLOS RÁPIDOS O DE CLÍNICA ATÍPICA}

Se trata de valorar si el consumo de cannabis repercute en la aparición de ciclos rápidos durante el seguimiento o la presencia de más clínica atípica. La mayoría de los trabajos no lo valoran. En uno de ellos se observa una asociación con mayor presencia de ciclos rápidos (Strakowski et al. 2007).

\subsection{EFECTOS DEL CONSUMO DE CANNABIS EN LA RESISTENCIA AL TRATAMIENTO}

Se pretende valorar la influencia del consumo de cannabis en la resistencia al tratamiento durante el seguimiento en el TB. No se valora en los estudios revisados. 


\begin{tabular}{|c|c|c|c|c|c|}
\hline Autor (año) & Muestra & $\begin{array}{l}\text { Tiempo } \\
\text { seguimiento }\end{array}$ & $\begin{array}{l}\text { Diagnóstico } \\
\text { consumo } \\
\text { cannabis }\end{array}$ & $\begin{array}{l}\text { Diagnóstico } \\
\text { trastorno } \\
\text { bipolar }\end{array}$ & Instrumentos y variables dependientes \\
\hline $\begin{array}{l}\text { Strakowski et } \\
\text { al. (2007) }\end{array}$ & $\begin{array}{l}144 \text { TB tipo I sin hospitalizaciones } \\
\text { previas ( } 33 \text { consumidores de } \\
\text { cannabis previo al inicio del TB; } 36 \\
\text { con inicio de TB antes que cannabis, } \\
48 \% \text { de consumidores) (elegibles } \\
\text { 172, pero } 28 \text { tenían menos de } 4 \\
\text { meses de seguimiento). }\end{array}$ & $\begin{array}{l}60 \text { meses. } \\
\text { Media de } \\
\text { seguimiento } \\
\text { fue } 2.6 \text { años. }\end{array}$ & $\begin{array}{l}\text { Módulo } \\
\text { Substance Use } \\
\text { Disorders del } \\
\text { SCID. } \\
\text { ASI. }\end{array}$ & $\begin{array}{l}\text { SCID. } \\
\text { Criterios DSM- } \\
\text { IV. } \\
\text { YMRS mayor } \\
\text { de } 20 \text {. }\end{array}$ & $\begin{array}{l}\text { Escalas YMRS. HAMD- } 17 \text {. } \\
\text { Recuperación (al menos } 8 \text { semanas seguidas con gravedad } \\
\text { de síntomas de } 102 \text { sobre } 6 \text { ). } \\
\text { Recurrencia (al menos una semana de puntuación superior a } \\
\text { 3). } \\
\text { Porcentaje de tiempo durante el seguimiento con síntomas } \\
\text { afectivos y de consumo (valoran tiempo en episodios } \\
\text { afectivos o de consumo evaluando cada } 4 \text { meses, valoran } \\
\text { semana por semana el mes previo de presencia de síntomas } \\
\text { afectivos o de consumo). }\end{array}$ \\
\hline $\begin{array}{l}\text { Van Rossum et } \\
\text { al. (2009) }\end{array}$ & $\begin{array}{l}3459 \text { TB tipo I, ingresados y } \\
\text { ambulatorios, iniciaron tratamiento } \\
\text { tras manía aguda. Iniciaron } 3684 \\
\text { sujetos, pero excluidos } 225 \text { por no } \\
\text { cumplir criterios de gravedad de } \\
\text { manía, además } 33 \text { excluidos por no } \\
\text { tener datos sobre consumo de } \\
\text { cannabis. Después de } 12 \text { semanas } \\
\text { iniciales quedaron } 1891 \text { pacientes. } \\
\text { En la visita basal hay un } 54.5 \% \text { de } \\
\text { consumidores de cannabis, parece } \\
\text { que hacen referencia a cualquier uso } \\
\text { de la sustancia, } 27.5 \% \text { con criterios } \\
\text { de abuso y } 6.8 \% \text { con criterios de } \\
\text { dependencia de cannabis. }\end{array}$ & $\begin{array}{l}12 \text { meses. El } \\
\text { estudio se } \\
\text { diseñó para } \\
24 \text { meses, } \\
\text { pero ofrecen } \\
\text { datos de los } \\
\text { primeros } 12 \\
\text { meses. }\end{array}$ & $\begin{array}{l}\text { Autoinformes } \\
\text { basales y durante } \\
\text { seguimiento, } \\
\text { valoran uso, } \\
\text { abuso, } \\
\text { dependencia } \\
\text { como acumulativo } \\
\text { durante el } \\
\text { seguimiento. }\end{array}$ & $\begin{array}{l}\text { Criterios CIE- } \\
10, \text { DSM-IV o } \\
\text { diagnóstico } \\
\text { clínico. }\end{array}$ & $\begin{array}{l}\text { CGI-BP global, de manía, de síntomas psicóticos y de } \\
\text { depresión (escala de } 7 \text { puntos de gravedad para cada } \\
\text { dimensión). } \\
\text { Cumplimiento. Variables de funcionamiento social } \\
\text { cualitativas. }\end{array}$ \\
\hline
\end{tabular}




\begin{tabular}{|c|c|c|c|c|c|}
\hline $\begin{array}{l}\text { Kim et al. } \\
(2015)\end{array}$ & $\begin{array}{l}239 \text { TB tipo I y esquizoafectivos ( } 5 \\
\text { sin datos sobre consumo, analizan } \\
234) .25 \text { consumidores (10.7\%). }\end{array}$ & 24 meses. & $\begin{array}{l}\text { Consumo de } 3 \\
\text { veces o más por } \\
\text { semana durante } \\
\text { los últimos tres } \\
\text { meses por } \\
\text { entrevista basal. }\end{array}$ & MINI. & $\begin{array}{l}\text { Número de remisiones por HAMD-21 (puntuación menor o } \\
\text { igual de 8) e YMRS (puntuación menor o igual de 12) } \\
\text { obtenidas en las } 9 \text { evaluaciones realizadas durante el } \\
\text { seguimiento. }\end{array}$ \\
\hline $\begin{array}{l}\text { Kvitland et al. } \\
(2015)\end{array}$ & $\begin{array}{l}62 \text { TB tipo I, primer tratamiento, } \\
\text { ingresados y ambulatorios. Incluyen } \\
101 \text { pacientes, pero } 62 \text { realizan el } \\
\text { seguimiento. } 6 \text { pacientes con } \\
\text { consumo continuado (al inicio y final } \\
\text { del seguimiento), } 7 \text { con consumo en } \\
\text { la valoración basal pero no en el } \\
\text { seguimiento y } 2 \text { con consumo } \\
\text { durante el seguimiento, pero no } \\
\text { basal. }\end{array}$ & 12 meses. & $\begin{array}{l}\text { Entrevista sobre } \\
\text { consumo. } \\
\text { Cualquier } \\
\text { consumo durante } \\
\text { el seguimiento. } \\
\text { Refirieron } \\
\text { consumo de 2-3 } \\
\text { veces por semana } \\
\text { de media. }\end{array}$ & $\begin{array}{l}\text { Criterios DSM- } \\
\text { IV. } \\
\text { SCID. }\end{array}$ & $\begin{array}{l}\text { YMRS. Funcionamiento por GAF-F (recuperación funcional } \\
\text { como puntuación de } 61 \text { ). } \\
\text { IDS-C. PANSS, PAS. } \\
\text { Recuperación sintomática como falta de episodios afectivos y } \\
\text { ausencia de síntomas psicóticos en } 6 \text { meses previos. }\end{array}$ \\
\hline $\begin{array}{l}\text { Zorrilla et al. } \\
\text { (2015) }\end{array}$ & $\begin{array}{l}1922 \text { TB tipo I (procedente de } \\
\text { estudio europeo EMBLEM que } \\
\text { incluyó } 3684 \text { pacientes, igual que } \\
\text { estudio de Van Rossum, de estos } \\
2416 \text { era elegibles por haberse } \\
\text { incluido en fase de mantenimiento } \\
\text { tras } 12 \text { primeras semanas). } \\
6.9 \% \text { consumidores activos, } 4.6 \% \\
\text { consumidores previos, } 88.5 \% \text { nunca } \\
\text { consumidores. }\end{array}$ & 24 meses. & $\begin{array}{l}\text { Autoinformes. } \\
\text { Previo uso: } \\
\text { cualquier } \\
\text { consumo entre } \\
\text { visita basal y } 12 \\
\text { semanas, } \\
\text { consumo actual } \\
\text { cualquier } \\
\text { consumo durante } \\
\text { seguimiento. }\end{array}$ & $\begin{array}{l}\text { Criterios CIE- } \\
10, \text { DSM-IV o } \\
\text { diagnóstico } \\
\text { clínico. }\end{array}$ & $\begin{array}{l}\text { CGI-BP, YMRS, HAMD-5. } \\
\text { Remisión (CGI-BP total menos de } 3 \text { en dos visitas } \\
\text { consecutivas y no recaída entre visitas). } \\
\text { Recurrencia (recaída tras haber obtenido la remisión). } \\
\text { Recuperación (remisión funcional y funcionamiento social } \\
\text { adecuado) durante el seguimiento. } \\
\text { Recaída (aumento puntuación CGI-BP desde visita previa). } \\
\text { Valoración funcional: deterioro laboral, relaciones, convivencia. }\end{array}$ \\
\hline
\end{tabular}

\section{Tabla 11. Descripción de los estudios de cohortes seleccionados.}

ASI: Addiction Severity Index; CGI-BP: Modification of the Clinical Global Impressions Scale for use in bipolar illness; GAF: Global Assessment of Functioning Scale; ; HAMD: Hamilton Depression Rating Scale; IDS-C: Inventory of Depression Scale; MINI: Mini-International Neuropsychiatric Interview.; PANSS: Positive and Negative Syndrome Scale; PAS: Premorbid Academic Functioning; SCID: Structured Clinical Interview for DSM Disorders; TB:Trastorno bipolar; YMRS: Young Mania Rating Scale. 


\begin{tabular}{|c|c|c|c|c|}
\hline Autor & Resultados principales & Otros hallazgos & Limitaciones & Observaciones \\
\hline $\begin{array}{l}\text { Strakowski et } \\
\text { al. (2007) }\end{array}$ & $\begin{array}{l}\text { Consumidores de cannabis } \\
\text { más tiempo en episodios } \\
\text { afectivos (maniacos y mixtos, } \\
\text { más el subgrupo de bipolar } \\
\text { primero) y más ciclos rápidos. } \\
\text { Grupo de cannabis primero } \\
\text { mejor recuperación, pero no } \\
\text { tras ajuste (por sexo, edad } \\
\text { inicio trastorno bipolar). No } \\
\text { diferencias en recurrencia. }\end{array}$ & $\begin{array}{l}\text { Remisión elevada de consumo tras } \\
\text { ingreso, pero recurrencia rápida. }\end{array}$ & Elevado porcentaje de alcohol. & $\begin{array}{l}\text { No diferencias en variables principales. } \\
\text { Múltiples comparaciones. }\end{array}$ \\
\hline $\begin{array}{l}\text { Van Rossum et } \\
\text { al. (2009) }\end{array}$ & $\begin{array}{l}\text { Menor cumplimiento y mayor } \\
\text { gravedad de la enfermedad, de } \\
\text { manía y psicosis. }\end{array}$ & $\begin{array}{l}\text { Menor satisfacción con la vida, menor } \\
\text { probabilidad de tener una relación. }\end{array}$ & $\begin{array}{l}\text { Muy elevado porcentaje de } \\
\text { consumidores de cannabis, parece } \\
\text { que se incluyen con cualquier } \\
\text { consumo, sólo } 11.3 \% \text { de no } \\
\text { consumidores se consideran en la } \\
\text { visita basal, lo que puede resultar en } \\
\text { menor discriminación de los } \\
\text { resultados. }\end{array}$ & $\begin{array}{l}\text { Alto porcentaje de consumo de alcohol y } \\
\text { otras drogas, aunque los resultados los } \\
\text { ajustan por estos. Un alto porcentaje de } \\
\text { sujetos se perdieron tras las primeras } 12 \\
\text { semanas de tratamiento. }\end{array}$ \\
\hline $\begin{array}{l}\text { Kim et al. } \\
\text { (2015) }\end{array}$ & $\begin{array}{l}\text { Menor remisión total en } \\
\text { consumidores (incluye HAMD y } \\
\text { YMRS). }\end{array}$ & $\begin{array}{l}\text { Menor remisión en escala HAMD en } \\
\text { mujeres. En varones, cannabis se } \\
\text { asoció con menor remisión en YMRS. } \\
\text { Remisión menor en consumidores de } \\
\text { cannabis y tabaco. }\end{array}$ & Sólo hay 9 mujeres consumidoras. & $\begin{array}{l}\text { No diferencias entre bipolares I y } \\
\text { esquizoafectivos. } \\
\text { Los datos de remisión se presentan } \\
\text { como mediana y rango intercuartil del } \\
\text { número de remisiones obtenidas durante } \\
\text { el seguimiento no como porcentaje de } \\
\text { sujetos que presentan remisión. }\end{array}$ \\
\hline $\begin{array}{l}\text { Kvitland et al. } \\
\text { (2015) }\end{array}$ & $\begin{array}{l}\text { Consumo continuado de } \\
\text { cannabis se asocia con manía } \\
\text { (mayor puntuación en escala } \\
\text { YMRS) y peor funcionamiento } \\
\text { (GAF) en análisis multivariante }\end{array}$ & $\begin{array}{l}\text { No diferencias en síntomas } \\
\text { depresivos o psicóticos. No } \\
\text { diferencias en remisión sintomática } \\
\text { durante el seguimiento ( } 50 \% \text { en } \\
\text { consumo continuado y } 68 \% \text { en no }\end{array}$ & $\begin{array}{l}\text { Sólo hay } 6 \text { pacientes con consumo } \\
\text { continuado. Los otros } 9 \\
\text { consumidores los incluyen en grupos } \\
\text { de no consumo continuado, lo que }\end{array}$ & $\begin{array}{l}\text { Las diferencias estadísticamente } \\
\text { significativas en análisis multivariante, } \\
\text { aunque no lo son en el bivariante } \\
\text { ( } p=0.06 \text { en el caso de la funcionalidad y }\end{array}$ \\
\hline
\end{tabular}




\begin{tabular}{|l|l|l|l|l|}
\hline & & $\begin{array}{l}\text { consumo continuado), ni en } \\
\text { recuperación funcional (67\% en } \\
\text { consumo continuado y 29\% en no } \\
\text { consumo continuado). }\end{array}$ & $\begin{array}{l}\text { puede atenuar las diferencias entre } \\
\text { grupos. } \\
\text { Resultados no estadísticamente } \\
\text { significativos por tamaño muestral en } \\
\text { análisis bivariante, significativos en } \\
\text { multivariante. }\end{array}$ & $\begin{array}{l}p=0.08 \text { en el caso de la puntuación de la } \\
\text { manía). }\end{array}$ \\
\hline $\begin{array}{l}\text { Zorrilla et al. } \\
(2015)\end{array}$ & $\begin{array}{l}\text { Consumidores previos tenían } \\
\text { mayores porcentajes de } \\
\text { remisión (68\%), recuperación } \\
(38.7 \%) \text { y menor porcentaje de } \\
\text { recurrencia (42\%) y recaída } \\
(29.8 \%) \text {. Evolución similar a } \\
\text { nunca consumidores. } \\
\text { Los consumidores activos } \\
\text { tenían menor recuperación y } \\
\text { remisión, mayor recurrencia y } \\
\text { mayor deterioro laboral y no } \\
\text { vivir con pareja en el modelo de } \\
\text { regresión logistica. }\end{array}$ & $\begin{array}{l}\text { Valoración del consumo por } \\
\text { autoinformes. Análisis posterior de } \\
\text { los resultados, no diseñado para este } \\
\text { objetivo. Criterios de resultado no } \\
\text { comparables con otros estudios. }\end{array}$ & $\begin{array}{l}\text { Más consumo de alcohol y otras drogas } \\
\text { en grupos de no consumidores de } \\
\text { alcohol. }\end{array}$ \\
$\begin{array}{l}\text { Al ajustar por consumo de alcohol y } \\
\text { otras drogas, el efecto de cannabis sólo } \\
\text { se asocia con la recurrencia, no con el } \\
\text { resto de las variables de resultado. }\end{array}$ \\
\hline
\end{tabular}

Tabla 12. Principales resultados de los estudios de cohortes seleccionados. 


\section{DISCUSIÓN}

El objetivo del presente trabajo es revisar la bibliografía existente sobre la repercusión del consumo de cannabis en la evolución del TB, seleccionando aquellos estudios de cohortes de pacientes con TB en los que se ha valorado el efecto del consumo de cannabis, excluyendo los estudios de seguimiento de sujetos con otros consumos de drogas que no los diferencian de consumo de cannabis y aquellos estudios donde se estudian los efectos del consumo de cannabis en sujetos bipolares pero de manera transversal sin seguimiento. De esta forma se han seleccionado 5 artículos. Los datos de estos trabajos sugieren que el consumo de cannabis empeora el pronóstico de este trastorno.

El tamaño muestral, en general, es destacado, excepto el de Kvitland et al. (2015). El trabajo de Strakowski et al. (2007) tiene una muestra de 144 sujetos, pero diferencia entre el subgrupo de bipolar primero y cannabis primero, por lo que los subgrupos quedan pequeños, intentando valorar diferencias según la secuencia de aparición del trastorno, en línea de un trabajo previo de los autores que observaban diferencias con el consumo de alcohol en pacientes bipolares según fuera la secuencia de los trastornos (Strakowski et al. 2005).

El seguimiento realizado es entre 12 y 60 meses, aunque el seguimiento de 60 meses no fue igual para todos, dado que hubo bastante pérdidas.

Estos estudios también observan que el consumo de cannabis es altamente prevalente entre pacientes bipolares, siendo la segunda o tercera droga de mayor frecuencia de consumo en esta población (compite con el consumo de alcohol), con cifras muy superiores al consumo en población general. Son cifras similares a las obtenidas en otros estudios. En una revisión se indica que el consumo de cannabis a lo largo de la vida en pacientes bipolares es del $70 \%$ y un 30\% tienen criterios de abuso o dependencia en el caso de los bipolares tipo I (Bally et al. 2014). En el estudio NESARC, hay una prevalencia de $7.2 \%$ de trastorno por uso de cannabis en los últimos 12 meses entre bipolares, frente al $1.2 \%$ en población general y refiriendo que son cifras inferiores a la de otros estudios (Lev-Ran et al. 2013a). En otra muestra encuentran un $29.4 \%$ de trastorno por uso de cannabis en pacientes bipolares (Agrawal 
et al. 2011). El consumo además puede influir en las manifestaciones clínicas del trastorno e, incluso se sugiere un papel etiológico o, al menos, precipitante del trastorno dado que adelanta la edad de inicio del TB. El consumo de cannabis se ha asociado con la inducción de hipomanía (Marwaha et al. 2018) y manía (Gibbs et al. 2014, Tijssen et al. 2010, Henquet et al. 2006).

\subsection{REPERCUSIÓN DEL CONSUMO DE CANNABIS EN LA EVOLUCIÓN DEL TRASTORNO BIPOLAR}

En general, se observa que el consumo de cannabis suele asociarse con una serie de repercusiones negativas en la evolución del $\mathrm{TB}$, aunque no todos los estudios valoran la misma variable dependiente. El consumo se asocia con un menor porcentaje de remisión clínica (Kim et al. 2015), menor recuperación, menor remisión, mayor recurrencia y peor funcionamiento laboral (Zorrilla et al. 2015), peor funcionamiento global (Kvitland et al. 2015), menor cumplimiento con el tratamiento, mayor gravedad de la enfermedad y menos satisfacción con la vida (Van Rossum et al. 2009). Aunque en uno de los estudios no se encontró relación con la recuperación y la recurrencia, se asociaba con una mayor duración de los episodios afectivos y más ciclos rápidos (Strakowski et al. 2007). Además de la presencia de ciclos rápidos, el consumo de cannabis se ha asociado con una mayor gravedad de la manía y con la presencia de síntomas psicóticos durante la fase aguda (Van Rossum et al. 2009) y se asocia más con la presencia de manía (Kvitland et al. 2015) que con depresión (Van Rossum et al. 2009). Aunque algunos observaron una mayor asociación con la depresión cuando se ajustaba por el consumo de alcohol (Strakowski et al. 2007).

Otros trabajos centrados en muestras clínicas de pacientes bipolares asocian el consumo de cannabis más con los episodios maníacos que depresivos o con la presencia de ciclos rápidos o características atípicas del cuadro. En una revisión se concluye que el consumo de cannabis se asocia con la exacerbación de la manía e incrementaba 3 veces el riesgo de la aparición de primeros episodios maníacos (Gibbs et al. 2014). En otra revisión, el consumo de cannabis se asocia con una edad más precoz de la manía, mayor frecuencia tanto de fases depresivas como maníacas y, también, de más ciclos rápidos y fases mixtas, además de un peor cumplimiento con el tratamiento (Bally et al 2014). En una muestra de pacientes bipolares, el consumo 
se relacionó con más episodios maníacos (Baethge et al. 2005) y, en otro estudio de casos-controles, observan que el trastorno por uso de cannabis en bipolares se asocia con más episodios mixtos y mayor discapacidad (Agrawal et al. 2011) y con más síntomas psicóticos durante la fase aguda (Braga et al. 2012). En otra muestra de pacientes bipolares tipo I ingresados, un $16 \%$ tenían un trastorno por uso de cannabis y este se asociaba con episodios maníacos o mixtos, presencia de síntomas psicóticos y mayor consumo de alcohol, tabaco y otras drogas (Weinstock et al. 2016).

En el estudio NESARC, el consumo de cannabis en bipolares se asoció con una edad de inicio del TB más precoz, mayor número de episodios maníacos, depresivos e hipomaníacos y más ciclos rápidos, pero no había diferencias entre bipolares tipo I y II (Lev-Ran et al. 2013a).

En un estudio donde se valoran los efectos del consumo de cannabis sobre el estado de ánimo en pacientes bipolares durante 6 días, se observa que el consumo de cannabis se asocia con la producción de síntomas maníacos y, a su vez, el afecto positivo se relaciona con mayor consumo de cannabis, pero no con los síntomas depresivos (Tyler et al. 2015).

El seguimiento de un caso de TB y consumidor de cannabis durante dos años, también indica un incremento en el número de días hipomaníacos y descenso de los días con depresión en relación con el consumo (El-Mallakh y Brown, 2007).

En un seguimiento de 642 pacientes bipolares fumadores de tabaco, se observa que el consumo de cannabis también se asoció con una peor evolución, presentaban una edad de inicio del TB más precoz, más ingresos, mayor número de episodios maníacos, depresivos y de síntomas psicóticos. La relación con los síntomas depresivos y psicóticos desaparecía al ajustar por otros factores de confusión (Lagerberg et al. 2016).

No podemos extraer conclusiones sobre si el consumo de cannabis aumenta el riesgo de suicidio en estos estudios pues no se ofrece información. En una revisión sobre el tema, se considera que el consumo de cannabis es un factor de riesgo para las tentativas autolíticas en los pacientes bipolares, con un 15-42\% de tentativas entre los bipolares consumidores frente a un 3.6-42\% de tentativas en bipolares no consumidores (Leite et al. 2015). En un estudio de casos-controles, observan que el trastorno por uso de 
cannabis en bipolares se asocia con más tentativas suicidas (Agrawal et al. 2011) pero, en el estudio NESARC, el consumo de cannabis en bipolares no presentaba diferencias respecto al riesgo de suicidio (Lev-Ran et al. 2013a).

Respecto al cumplimiento y la adherencia al tratamiento con estos trabajos no se pueden establecer conclusiones definitivas pues uno no observa diferencias (Strakowski et al. 2007) y otro sí (Van Rossum et al. 2009), mientras que los demás no lo valoran. El consumo de cannabis, en general, se considera como un factor predictor de mala adherencia al tratamiento (González-Pinto et al. 2010).

Por lo tanto, el consumo de cannabis se asocia con peor recuperación del trastorno bipolar durante el seguimiento, peor funcionamiento global y, posiblemente, con mayor duración de los episodios afectivos, más presencia de ciclos rápidos y parece asociarse más con fases maníacas que depresivas.

\subsection{CARACTERÍSTICAS BASALES DIFERENCIALES ENTRE LOS CONSUMIDORES Y NO DE CANNABIS}

No está claro si los consumidores de cannabis presentan una mayor gravedad del trastorno en el momento de la valoración. Había diferencias entre los distintos estudios.

El trabajo de Kim et al. (2015) encuentra que los consumidores tenían mayor gravedad inicial, mayor puntuación en la escala de manía y depresión, aunque no había diferencias en la edad de inicio del TB, ni diferencias en características clínicas como la ideación suicida o la presencia de síntomas psicóticos. Kvitland et al. (2015) no encuentran diferencias en la presencia de síntomas depresivos o psicóticos.

Strakowski et al. (2009) diferencian entre los pacientes bipolares consumidores de cannabis dos subgrupos, aquellos donde el TB se manifiesta primero (bipolar primero) de los que presentan el consumo antes que el TB (cannabis primero), siendo la edad de inicio del TB distinto en los dos grupos (más tardía en subgrupo de cannabis primero). En los consumidores había más presencia de síntomas psicóticos basal pero no había diferencias en la puntuación de las escalas de depresión o manía ni diferencias en la presencia de episodios mixtos. 
Van Rossum et al. (2009) observan un inicio más precoz del TB en los consumidores (24 años frente 31 años), mayor gravedad inicial en la valoración de manía y síntomas psicóticos y había un mayor porcentaje de ingresados frente a pacientes ambulatorios. Zorrilla et al. (2015) con un estudio basado en la misma cohorte que el de Van Rossum et al. (2009), observan que los consumidores de cannabis activos tienen mayor número de episodios maniacos previos, mayor insatisfacción con la vida, mayor gravedad global y de la manía, más presencia de cicladores rápidos y mayor frecuencia de intentos de suicidio previos. Ambos grupos de consumidores tenían mayor frecuencia de pacientes ingresados, menor proporción de sujetos viviendo con pareja, más síntomas psicóticos, más intentos de suicidio, una edad de inicio del TB más precoz, más ingresos previos y peor adherencia a la medicación. No había diferencias en la presencia de síntomas depresivos.

Por lo tanto, aunque la mayoría observan una edad de inicio del TB más precoz entre los consumidores, no analizan su efecto como posible factor pronóstico en las repercusiones del consumo de cannabis sobre el $\mathrm{TB}$, dado que la edad de inicio precoz del TB puede ser un factor de mal pronóstico (Grande et al. 2016).

En general el consumo de cannabis se presenta como un posible precipitante del TB y se suele encontrar una edad de inicio de este trastorno más precoz en los consumidores (Lagerberg et al. 2016). En una revisión se señala que el primer episodio maníaco se presenta de media a los 19.5 años entre los consumidores y de 25 años entre los no consumidores mientras que, para la primera fase depresiva, es de 18.5 y 24.4 años respectivamente (Leite et al. 2015). Este inicio más precoz entre los consumidores también es habitual para la psicosis (González-Pinto et al. 2008).

\subsection{INFLUENCIA DE LAS CARACTERÍSTICAS SOCIODEMOGRÁFICAS EN EL EFECTO DEL CONSUMO SOBRE LA EVOLUCIÓN DEL TRASTORNO BIPOLAR}

En todos los trabajos los consumidores son más jóvenes y presentan predominio de varones, por lo que al haber pocas mujeres consumidoras no suele realizarse este análisis. Los trabajos no valoran la edad basal como posible predictor de la evolución. Tampoco suele haber análisis por sexo. El único que lo analiza es el trabajo de Kim et 
al. (2015) que señalan que las mujeres consumidoras tienen menor remisión, pero sólo hay 9 mujeres para el análisis, por lo que no pueden extraerse conclusiones al respecto. Sería importante valorar el efecto de la edad y el sexo como factores predictores, pues pueden tener distintos cursos evolutivos. En un estudio entre sujetos con un primer episodio maníaco, los varones se asociaban con una mayor presencia de TUS y peor funcionamiento y mayor gravedad del cuadro, mientras que las mujeres se asociaban con antecedentes de abuso sexual (Cotton et al. 2013).

\subsection{INFLUENCIA DE LAS CARACTERÍSTICAS DEL TRASTORNO BIPOLAR EN LOS EFECTOS DEL CONSUMO DE CANNABIS EN LA EVOLUCIÓN}

La mayoría de los trabajos valoran pacientes con TB tipo I, generalmente a partir de un primer episodio maníaco. Kim et al. (2015) incluyen también pacientes con trastorno esquizoafectivo tipo bipolar y no observan diferencias evolutivas. Por lo tanto, no tenemos datos sobre los efectos del consumo de cannabis en otros subtipos de TB como el tipo II.

En general, tampoco valoran la influencia de la gravedad inicial del TB en la evolución posterior, ni se valora la repercusión del tipo de fases predominantes previas o basales (maniacas, depresivas, mixtas) aunque, en general, se valoran los sujetos a partir de una fase de manía inicial.

Kvitland et al. (2016) en un subanálisis de los datos de la cohorte que hemos mencionado (Kvitland et al. 2015) observan que la duración de TB no tratado podía relacionarse con el consumo de cannabis posterior.

Por lo tanto, no podemos extraer conclusiones sobre si las características del TB son relevantes en los efectos del consumo de cannabis.

\subsection{INFLUENCIA DE LAS CARACTERÍSTICAS DEL CONSUMO DE CANNABIS EN LOS EFECTOS DEL CANNABIS EN LA EVOLUCIÓN}

Los criterios de consumo son dispares entre los distintos estudios y no comparables. La definición de consumo en el trabajo de Kim et al. (2015) es de consumo 3 o más días por semana, mientras que Strakowski et al. (2007) si utilizan los criterios 
establecidos de abuso o dependencia. La prevalencia de consumidores en las muestras es muy variable, un $10.7 \%$ de consumidores en la muestra de Kim et al. (2015), o un 47.9\% de abuso-dependencia en la de Strakowski et al. (2007). Kvitland et al. (2015) valoran por autoinformes la presencia de cualquier consumo. Zorrilla establecen los criterios de uso, abuso y dependencia por autoinformes, pero para el análisis lo agrupan y no diferencian los posibles efectos por separado, al igual que Van Rossum. Estos últimos también valoran el consumo durante el seguimiento y suman la gravedad, pero no ofrecen datos sobre las repercusiones en la evolución o sobre las repercusiones de mantener el consumo. La mayoría no presentan datos sobre cantidad de consumo, frecuencia o potencia del cannabis.

Por lo tanto, sería recomendable homogeneizar los criterios de consumo de cannabis y serían necesarios estudios con muestras más grandes para valorar la influencia de la gravedad de la dependencia en la evolución. Es esperable que los dependientes de cannabis tengan una evolución más negativa que los consumidores sin criterios de dependencia, pero estos trabajos los han agrupado en consumidores y no consumidores y no han realizado análisis de estos subgrupos. Posiblemente la mayor potencia del cannabis puede también repercutir en el riesgo de recaída del TB.

De datos del estudio NESARC, se observa que los pacientes bipolares consumidores de cannabis consumen más cantidad y con más frecuencia cannabis que los sujetos consumidores con depresión (Taub et al. 2018), pero no hay diferencias en la edad de inicio en el consumo de cannabis entre bipolares y no (Lev-Ran et al. 2013a).

La edad de inicio en el consumo tampoco se valora como factor pronóstico en ninguno de los estudios analizados. Strakowski et al. (2007) diferencian el subgrupo de cannabis primero con edad de inicio a los 16 años y los bipolares primero con edad de inicio a los 18 años, aunque la diferencia no es estadísticamente significativa y no valoran el efecto de la edad de inicio en la evolución. En general, la edad de inicio en el consumo se considera relevante en la evolución de la dependencia de cannabis y en las consecuencias asociadas. 


\subsection{EFECTOS DEL POLICONSUMO EN LOS EFECTOS DEL CONSUMO DE CANNABIS SOBRE EL TRASTORNO BIPOLAR}

Dado que es habitual el patrón de policonsumo, es importante considerar los posibles efectos del consumo de otras drogas. El consumo de cannabis se asocia principalmente con el consumo de tabaco (la gran mayoría) y de alcohol. Las cifras de comorbilidad entre estos consumos en los estudios son muy elevadas y, en general, se observa que la presencia de otros consumos medía en algunos de los efectos negativos del consumo de cannabis sobre la evolución. En el estudio NESARC, la prevalencia de otros trastornos por uso de sustancias entre los adictos al cannabis con TB era de 67\% para el tabaco, 66\% para el alcohol y 71\% para otras drogas (Lev-Ran et al. 2013a).

Los consumos de drogas en general influyen negativamente en la evolución del TB. Así el consumo de alcohol se ha observado que tiene relevancia (Strakowski et al. 2005), por lo que es importante controlar el efecto de estos consumos.

Kvitland et al. (2015) no ofrecen datos sobre el policonsumo. Kim et al. (2015) ofrecen datos sobre el consumo de alcohol y tabaco, pero, de forma sorprendente, no hay diferencias significativas en el porcentaje de abuso o dependencia de alcohol entre consumidores y no de cannabis, posiblemente por el tamaño muestral. Tampoco observan diferencias en la evolución del TB debido al consumo de alcohol, pero sólo hay 8 sujetos con abuso o dependencia de alcohol entre los 25 consumidores de cannabis. Por el contrario, encuentran que la presencia concomitante de consumo de tabaco y cannabis se asocia con una menor remisión.

Zorrilla et al. (2015) encuentran que los consumidores de alcohol se distribuyen en un $37.7 \%$ entre los consumidores de cannabis actual, un $13.4 \%$ en consumidores de cannabis previo y un $49 \%$ entre los no consumidores de cannabis. Otras drogas se distribuyen en un $3.7 \%$ entre los consumidores de cannabis actual, un $4.4 \%$ entre los consumidores de cannabis previo y un $91.9 \%$ entre los no consumidores, lo que es sorprendente, dado que es lo contrario a lo esperable por la literatura. No ofrecen datos sobre los tipos de drogas que consumen. El consumo de alcohol y otras drogas tiene efectos negativos en la evolución funcional y se asocian con menor recuperación y remisión. En el análisis multivariante ajustado por el efecto de estas drogas, el efecto de cannabis en la evolución se atenúa, sobre todo en la evolución funcional. El 
consumo actual de cannabis se asocia sólo con una mayor recurrencia en este modelo multivariante donde se incluyen el consumo de otras drogas.

Van Rossum et al. (2009), con datos de la misma cohorte que el estudio de Zorrilla et al. (2015), valoran igualmente consumo de alcohol y otras drogas en la valoración basal y en el seguimiento. Diferencian uso, abuso y dependencia de estas drogas, pero no tienen en cuenta la diferencia de gravedad para el análisis de los resultados. Los consumidores de cannabis presentan un mayor consumo de alcohol y de otras drogas. Hay un $80.3 \%$ de consumo de alcohol basal en los consumidores de cannabis y un $37.2 \%$ entre los no consumidores y un $34.3 \%$ de consumo de otras drogas entre los consumidores de cannabis y un $2.6 \%$ entre los no consumidores, ambos porcentajes disminuyen durante el seguimiento. El consumo de otras drogas modifica los efectos del consumo de cannabis sobre el cumplimiento del tratamiento y la satisfacción con la vida.

Strakowski et al. (2007) indican que un 60\% del subgrupo de bipolar primero tienen abuso o dependencia de alcohol y un $70 \%$ del subgrupo de cannabis primero, además más graves en este último, pero incluso en el subgrupo de no consumidores de cannabis, un 22\% tenían abuso o dependencia de alcohol. Al ajustar el análisis por el consumo de alcohol, el consumo de cannabis se asocia con la duración de la depresión, con menos tiempo en remisión afectiva y presencia de síntomas subsindrómicos. El elevado porcentaje de consumo de alcohol puede influir en los resultados. Respecto a otras drogas, no había en el grupo de no consumidores de cannabis y había 11 en los otros dos subgrupos, un $8 \%$ de los bipolares primeros y un $24 \%$ del subgrupo de cannabis primero, pero no valoran la asociación con la evolución.

Estos autores previamente observaron que, en el caso del consumo de alcohol, los pacientes con consumo de alcohol previo al TB se recuperaban más, mientras que los sujetos con consumo de alcohol posterior al TB presentaban más tiempo en episodios afectivos y tenían mayor consumo de alcohol. Es decir, parece que el efecto de la secuencia de los trastornos era más relevante para el alcohol que para el cannabis. Al igual que con el cannabis, observaban que tras el ingreso por manía disminuía el consumo de alcohol, pero con una elevada recurrencia posterior (Strakowski et al. 2005). 
En un seguimiento de pacientes bipolares I ingresados por manía con consumo de tabaco, se observó que el tabaco no predecía un peor curso del TB, pero si incrementaba el riesgo de consumo de cannabis y alcohol en el subgrupo de adolescentes (Heffner et al. 2012).

Otros autores han señalado que el consumo de cannabis se asocia con la manía, mientras que el de alcohol con la depresión (Strakowski et al. 2000) y en un seguimiento de dos años (Baethge et al. 2005).

Por lo tanto, el consumo de otras drogas son factores de confusión que hay que considerar cuando se evalúan los efectos del consumo de cannabis en la evolución del TB. Sería interesante valorar muestras con consumidores de cannabis exclusivamente, dado que el elevado número de sujetos con otros consumos modifican los resultados.

\subsection{EFECTOS DEL CESE DEL CONSUMO EN LA EVOLUCIÓN DEL TRASTORNO BIPOLAR}

La persistencia en el consumo durante el seguimiento no lo valora el estudio de Kim et al. (2015) y otros autores valoran la persistencia en el consumo, pero no analizan el efecto (Van Rossum et al. 2009).

Strakowski et al. (2007) observan que un 70\% de los consumidores se recuperan del consumo tras el ingreso por manía, pero se produce un $60 \%$ de recurrencia en el consumo posterior, lo que sugieren que es una época adecuada para la intervención terapéutica.

En otro estudio, el objetivo principal del trabajo es valorar el efecto de la persistencia en el consumo de cannabis sobre la evolución. El mantenimiento del consumo se asocia con una mayor puntuación en la escala de manía y peor funcionamiento, aunque no estadísticamente significativo (Kvitland et al. 2015).

El objetivo principal del trabajo de Zorrilla et al. (2015) es también valorar el efecto del cese del consumo de cannabis en la evolución del TB. Señalan que los consumidores de cannabis previo al seguimiento tenían mayores porcentajes de remisión, recuperación y menor porcentaje de recurrencia y recaída que los consumidores activos durante el seguimiento, con una evolución similar a los nunca consumidores. Los consumidores activos tenían mayor deterioro laboral, aunque estos 
efectos eran menos manifiestos cuando se ajustaba por el efecto de otras drogas. Por lo tanto, los datos sugieren los beneficios del cese del consumo durante el seguimiento. El cese del consumo de cannabis también se ha visto que mejora el pronóstico en los pacientes con psicosis mejorando su funcionamiento (González-Pinto et al. 2011) y en otro estudio, el cese tras un primer ingreso por psicosis mejoraba la gravedad clínica al año de seguimiento (Stone et al. 2014).

\subsection{EFECTOS COGNITIVOS DEL CONSUMO DE CANNABIS DURANTE EL SEGUIMIENTO DEL TRASTORNO BIPOLAR}

Se trata de valorar si el consumo de cannabis repercute en la evolución del deterioro neuropsicológico en los sujetos con TB, pero no lo valora ninguno de los estudios revisados. Es un tema controvertido, dado que los datos de otros estudios son discrepantes. Así se ha señalado que los sujetos bipolares con consumo de cannabis se encuentran mejor cognitivamente (Braga et al. 2012) y, así se concluye en una revisión reciente (Bally et al. 2014). Estos autores señalan como posibles explicaciones la necesidad de más habilidades cognitivas para obtener la droga o por el papel del cannabis como posible desencadenante del cuadro en sujetos inicialmente menos graves (Bally et al. 2014). Sin embargo, en otra revisión, el consumo de cannabis en el TB se relacionaba con dificultades en la toma de decisiones, en la memoria de trabajo y el tiempo de reacción (Cahill et al. 2006).

\subsection{EVALUAR LA REPERCUSIÓN DEL CONSUMO EN LA PRESENCIA DE CICLOS RÁPIDOS Y MANIFESTACIONES CLÍNICAS ATÍPICAS}

Se trata de valorar si el consumo de cannabis repercute en la aparición de ciclos rápidos durante el seguimiento o la presencia de clínica atípica. La mayoría de los trabajos no lo valoran. Como hemos indicado, en uno de ellos se observa una asociación con mayor presencia de ciclos rápidos (Strakowski et al. 2007). Por lo tanto, no podemos obtener conclusiones sobre el tema. 


\subsection{EFECTOS DEL CONSUMO DE CANNABIS EN LA RESISTENCIA AL TRATAMIENTO}

En los estudios analizados no valoran la relación con la resistencia al tratamiento del TB. Existe muy poca literatura al respecto, por lo que sería interesante analizarlo en estudios posteriores.

Como repercusiones prácticas relevantes del trabajo se puede señalar que el cese del consumo mejora el pronóstico, el cese en el consumo se asocia con una evolución similar a la de los no consumidores o puede que incluso mejor, por lo que hay que valorar siempre en estos pacientes bipolares la presencia de dicho consumo e intervenir sobre este, pues va a mejorar el pronóstico. Además, tras un primer ingreso por manía hay que realizar una intervención terapéutica sobre dicho consumo dado que es un momento ideal para el cese e intentar mantener la abstinencia para mejorar la evolución.

\section{LIMITACIONES}

Como limitaciones del presente trabajo hay que indicar que pueden existir trabajos que no se hayan encontrado, dado que la búsqueda ha sido sólo en Pubmed. Por otro lado, en los trabajos obtenidos existe una elevada heterogeneidad en las medidas evolutivas, con distintos criterios de remisión, recurrencia, etc. Igualmente existe una amplia heterogeneidad en la medida de consumo de cannabis. La metodología utilizada para valorar los resultados es diferente entre los distintos trabajos. Son pocos trabajos de seguimiento por lo que los resultados no son concluyentes.

Se han seleccionado fundamentalmente pacientes bipolares tipo I, habitualmente en fase maníaca, en muchos casos ingresados, por lo que se han seleccionado pacientes más graves. Los datos pueden no ser extrapolables para pacientes menos graves e, igualmente no hay datos sobre pacientes bipolares tipo II.

Existen pocos datos sobre cómo influyen las características o gravedad del consumo, las características del TB o las características sociodemográficas en el efecto del consumo de cannabis en la evolución del TB, por lo que no se pueden extraer conclusiones al respecto. 


\section{CONCLUSIONES}

El consumo de cannabis tiene un efecto negativo sobre la recuperación y la funcionalidad del TB durante el seguimiento.

El cese del consumo mejora el pronóstico y la evolución del TB.

El consumo concomitante de otras drogas son factores de confusión que hay que tener en cuenta a la hora de analizar los resultados y de diseñar nuevos trabajos.

No hay información sobre la influencia de las características del TB o las características del consumo de cannabis en los efectos del consumo de cannabis sobre la evolución del TB. 


\section{BIBLIOGRAFÍA}

Agrawal, A., Nurnberger, J.I. y Lynskey, M.T. (2011). Cannabis involvement in individuals with bipolar disorder. Psychiatry Research, 185, 459-461.

American Psychiatric Association (APA), (2002). Manual Diagnóstico y Estadístico de los Trastornos Mentales DSM-IV-TR. Barcelona, Masson.

American Psychiatric Association (APA), (2015). Manual Diagnóstico y Estadístico de los Trastornos Mentales DSM-5. 5a edición. Barcelona, Masson.

Angst, J. y Marneros, A. (2001). Bipolarity from ancient to modern times: conception, birth and rebirth. Journal of Affective Disorders, 67:3-19.

Arendt, M., Rosenberg, R., Foldager, L., Perto, G. y Munk-Jørgensen, P. (2007). Psychopathology among cannabis-dependent treatment seekers and association with later substance abuse treatment. Journal of Substance Abuse and Treatment, 32, 113 119.

Ashton, C.H., Moore, P.B., Gallagher, P. y Young, A.H. (2005). Cannabinoids in bipolar affective disorder: a review and discussion of their therapeutic potential. Journal of Psychopharmacology, 19, 293-300.

Baethge, C., Baldessarini, R.J., Khalsa, H.M., Hennen, J., Salvatore, P. y Tohen, M. (2005). Substance abuse in first-episode bipolar I disorder: indications for early intervention. American Journal of Psychiatry, 162:1008-1010.

Bally, N., Zullino, D. y Aubry, J.M. (2014). Cannabis use and first manic episode. Journal of Affective Disorders, 165:103-108. doi: 10.1016/j.jad.2014.04.038.

Bobes, J. y Calafat, A. (ed). (2000). Monografía Cannabis. Adicciones, 12, suplemento 2.

Bourgeois, M.L. y Verdoux, H. (1997). Trastornos bipolares del estado de ánimo. Barcelona, Masson.

Braga, R.J.., Burdick, K.E., Derosse, P.y Malhotra, A.K. (2012) Cognitive and clinical outcomes associated with cannabis use in patients with bipolar I disorder. Psychiatry Research, 200:242-245. doi: 10.1016/j.psychres.2012.05.025. 
Cahill, C.M., Malhi, G.S., Ivanovski, B., Lagopoulos, J. y Cohen, M. (2006). Cognitive compromise in bipolar disorder with chronic cannabis use: cause or consequence? Expert Review Neurotherapeutics, 6:591-8. doi: 10.1586/14737175.6.4.591

Cassidy, F., Ahearn, E.P., Carroll, B.J. (2001). Substance abuse in bipolar disorder. Bipolar Disorders, 3:181-8.

Cerullo, M. A. y Strakowski, S.M. (2007). The prevalence and significance of substance use disorders in bipolar type I and II disorder. Substance Abuse Treatment, Prevention and Policy, 1; 2:29.

Cotton, S.M., Lambert, M., Berk, M., Schimmelmann, B.G., Butselaar, F.J., McGorry, P.D. y Conus, P. (2013). Gender differences in first episode psychotic mania. BMC Psychiatry 13:82.

Diagnostic and Statistical Manual of Mental Disorders. (1994). 4. ${ }^{\mathrm{a}} \mathrm{ed}$. Washington DC: American Psychiatric Association.

De Dios, C., Goikolea, J.M., Colom, F., Moreno, C., Vieta, E. (2014). Los trastornos bipolares en las nuevas clasificaciones: DSM-5 y CIE-11. Revista de Psiquiatría y Salud Mental, 7: 179-185.

El-Mallakh, R.S., Brown, C. (2007). The effect of extreme marijuana use on the longterm course of bipolar I illness: a single case study. Journal of Psychoactive Drugs, 39:201-202.

Gibbs, M., Winsper, C., Marwaha S., Gilbert, E., Broome, M. y Singh, S.P. (2014). Cannabis use and mania symptoms: a systematic review and meta-analysis. Journal of Affective Disorders.171:39-47. doi: 10.1016/j.jad.2014.09.016.

Goikolea, J.M. y Vieta, E. (2012). Protocolos de intervención en patología dual. Trastorno bipolar. Brainpharma. EdikaMed, S.L. Barcelona.

Goldberg, J.F., Garno, J.L., Leon, A.C., Kocsis, J.H. y Portera, L. (1999). A history of substance abuse complicates remission from acute mania in bipolar disorder. Journal of Clinical Psychiatry. 60:733-40.

González-Pinto, A., Alberich, S., Barbeito, S., Alonso, M., Vieta, E., et al. (2010). Different profile of substance abuse in relation to predominant polarity in bipolar 
disorder: the Vitoria long-term follow-up study. Journal of Affective Disorders, 124:250-255.

González-Pinto, A., Alberich, S., Barbeito, S., Gutierrez, M., Vega, P., Ibáñez, B., ... Arango, C. (2011). Cannabis and first-episode psychosis: different long-term outcomes depending on continued or discontinued use. Schizophrenia Bulletin. 37:631-9. doi: 10.1093/schbul/sbp126.

González-Pinto, A., Ballesteros, J., Aldama, A., Pérez de Heredia, J.L., Gutiérrez, M. y Mosquera, F. (2003). Principal components of mania. Journal of Affective Disorders, 76:95-102.

González-Pinto, A., Mosquera, F., Alonso, M., López, P. y Ramírez, F. (2006). Suicidal risk in bipolar I disorder patients and adherence to long-term lithium treatment. Bipolar Disorders, 8:618-624.

González-Pinto, A., Reed, C., Novick, D., Bertsch, J. y Haro, J.M. (2010). Assessment of medication adherence in a cohort of patients with bipolar disorder. Pharmacopsychiatry, 43:263-270. doi: 10.1055/s-0030-1263169.

González-Pinto, A., Vega, P., Ibáñez, B., Mosquera, F., Barbeito, S., Gutiérrez, M., ... Vieta, E. (2008). Impact of cannabis and other drugs on age at onset of psychosis. Journal of Clinical Psychiatry, 69:1210-1216.

Grande, I., Berk, M., Birmaher, B., Vieta, E. (2016). Bipolar disorder. Lancet. 9; 387:1561-1572. doi: 10.1016/S0140-6736(15)00241-X.

Grant, B.F., Stinson, F.S., Hasin, D.S., Dawson, D.A. y Chou, S.P. (2005). Prevalence, correlates, and comorbidity of bipolar I disorder and axis I and II disorders: results from the National Epidemiologic Survey on Alcohol and Related Conditions. Journal of Clinical Psychiatry, 66:1205-15.

Heffner, J.L., DelBello, M.P., Anthenelli, R.M., Fleck, D.E., Adler, C.M. y Strakowski, S.M. (2012). Cigarette smoking and its relationship to mood disorder symptoms and co-occurring alcohol and cannabis use disorders following first hospitalization for bipolar disorder. Bipolar Disorders, 14:99-108. doi: 10.1111/j.1399-5618.2012.00985. x. 
Henquet, C., Krabbendam, L., de Graaf, R., ten Have, M. y van Os, J. (2006). Cannabis use and expression of mania in the general population. Journal of Affective Disorders, 95, 103-110.

Informe de la Comisión Clínica de la Delegación del Gobierno para el Plan Nacional sobre Drogas. (2006). Informe sobre cannabis. Madrid: Plan Nacional sobre Drogas.

Kessler, R.C., Crum, R.M., Warner, L.A., Nelson, C.B. y Schulenberg, J. (1997). Lifetime co-occurrence of DSM-III-R alcohol abuse and dependence with other psychiatric disorders in the National Comorbidity Survey. Archives of General Psychiatry, 54:313-21.

Kim, S-W., Dodd, S., Berk, L., Kulkarni, J., De Castella, A., Fitzgerald, P.B., ... Berk, M. (2015). Impact of Cannabis Use on Long-Term Remission in Bipolar I and Schizoaffective Disorder. Psychiatry Investigation, 12:349-355. doi:10.4306/pi.2015.12.3.349.

Kvitland, L.R., Melle, I., Aminoff, S.R., Demmo, C., Lagerberg, T.V., Andreassen, O.A.y Ringen, P.A. (2015). Continued cannabis use at one year follow up is associated with elevated mood and lower global functioning in bipolar I disorder. $B M C$ Psychiatry.5;15:11. doi: 10.1186/s12888-015-0389-x.

Kvitland, L.R., Ringen, P.A, Aminoff, S.R., Demmo, C., Hellvin, T., Lagerberg, T.V., Andreassen, O.A. y Melle, I. (2016). Duration of untreated illness in first-treatment bipolar I disorder in relation to clinical outcome and cannabis use. Psychiatry Research, 246: 762-768. doi: 101016/j.pyschrech.2016.07.064.

Lagerberg, T.V., Icick, R., Andreassen, O.A., Ringen, P.A., Etain, B., Aas, M., ... Bellivier, F. (2016). Cannabis use disorder is associated with greater illness severity in tobacco smoking patients with bipolar disorder. Journal of Affective Disorders, 15;190:286-293. doi: 10.1016/j.jad.2015.10.023.

Lagerberg, T.V., Sundet, K., Aminoff, S.R., Berg, A.O., Ringen, P.A., Andreassen, O.A.y Melle, I. (2011). Excessive cannabis use is associated with earlier age at onset in bipolar disorder. European Archives of Psychiatry and Clinical Neurosciences, 261, 397-405. 
Leite, R.T., Nogueira, S. O., do Nascimento, J.P., de Lima, L.S., da Nóbrega, T.B., Virgínio, M. S., ...Souza, F.G. (2015). The Use of Cannabis as a Predictor of Early Onset of Bipolar Disorder and Suicide Attempts. Neural Plasticity. 2015:434127. doi: $10.1155 / 2015 / 434127$.

Lev-Ran, S., Le Foll, B., McKenzie, K., George, T.P., y Rehm, J. (2013a). Bipolar disorder and co-occurring cannabis use disorders: characteristics, co-morbidities and clinical correlates. Psychiatry Research, 209, 459-465.

Lev-Ran, S., Le Foll, B., McKenzie, K., George, T.P. y Rehm, J. (2013b). Cannabis use and cannabis use disorders among individuals with mental illness. Comprehensive Psychiatry, 54, 589-598.

Marwaha, S., Winsper, C., Bebbington, P. y Smith, D. (2018). Cannabis Use and Hypomania in Young People: A Prospective Analysis. Schizophrenia Bulletin, 17:1267-1274. doi: 10.1093/schbul/sbx158.

Observatorio Europeo de las drogas y las toxicomanías. (2017). Informe Europeo sobre drogas 2017. Tendencias y novedades. Oficina de Publicaciones de la Unión Europea. Luxemburgo.

O.M.S. CIE-10 (1992). Trastornos Mentales y del Comportamiento. Décima Revisión de la Clasificación Internacional de las Enfermedades. Descripciones Clínicas y pautas para el diagnóstico. Ginebra: Organización Mundial de la Salud.

Prisciandaro, J.J., Mellick, W., Mitaro, E.y Tolliver, B.K. (2019). An evaluation of the impact of co-occurring anxiety and substance use disorder on bipolar disorder illness outcomes in STEP-BD. Journal of Affective Disorders, 246:794-799. doi: 10.1016/j.jad.2018.12.070.

Ramos, J.A. (coordinador). (2017). Instituto universitario de Investigación en Neuroquímica. Efectos terapéuticos de los cannabinoides. Madrid: Instituto universitario de Investigación en Neuroquímica de la Universidad Complutense de Madrid.

Regier, D.A., Farmer, M.E., Rae, D.S., Locke, B.Z. y Keith, S.J. (1990). Comorbidity of mental disorders with alcohol and other drug abuse. Results from the Epidemiologic Catchment Area (ECA) Study. JAMA. 264:2511-2518. 
Roffman, R.A. y Stephens, R.S. (ed). (2006). Cannabis dependence. Its nature, consequences and treatment. Cambridge: Cambridge University Press.

Stone, J.M., Fisher, H.L., Major, B., Chisholm, B., Woolley, J., Lawrence, J., ... Young, A.H.; MiData Consortium. (2014). Cannabis use and first-episode psychosis: relationship with manic and psychotic symptoms, and with age at presentation. Psychological Medicine, 44:499-506. doi: 10.1017/S0033291713000883.

Strakowski, S.M., DelBello, M.P., Fleck, D.E., Adler, C.M., Anthenelli, R.M. y Keck, P.E. Jr., (2005). Effects of co-occurring alcohol abuse on the course of bipolar disorder following a first hospitalization for mania. Archives of General Psychiatry, 62:851858.

Strakowski, S.M., DelBello, M.P., Fleck, D.E., Adler, C.M., Anthenelli, R.M., Keck, P.E. Jr., ... Amicone, J. (2007). Effects of co-occurring cannabis use disorders on the course of bipolar disorder after a first hospitalization for mania. Archives of General Psychiatry, 64:57-64.

Strakowski, S.M., DelBello, M.P., Fleck, D.E. y Arndt, S. (2000). The Impact of Substance Abuse on the Course of Bipolar Disorder. Biological Psychiatry 48:477485.

Taub, S., Feingold, D., Rehm, J. y Lev-Ran, S. (2018). Patterns of cannabis use and clinical correlates among individuals with Major Depressive Disorder and Bipolar Disorder. Comprehensive Psychiatry, 80:89-96. doi: 10.1016/j.comppsych.2017.09.004.

Tijssen, M.J., Van Os, J., Wittchen, H.U., Lieb, R., Beesdo, K. y Wichers, M. (2010). Risk factors predicting onset and persistence of subthreshold expression of bipolarpsychopathology among youth from the community. Acta Psychiatrica Scandinavica, 122:255-66. doi: 10.1111/j.1600-0447.2010.01539. x.

Tyler, E., Jones, S., Black, N., Carter, L.A. y Barrowclough, C. (2015). The relationship between bipolar disorder and cannabis use in daily life: an experience sampling study. PLoS One, 4;10: e0118916. doi: 10.1371/journal.pone.0118916.

Uribe, F. y Zorrilla, I. (2009). Trastorno bipolar. En: Manual del residente de psiquiatría. Madrid, Grupo ENE Life Publicidad, S.A. Pp: 295-302. 
Ustundag, M.F., Ozhan, E., Yucel, A. y Ozcan, H. (2015). Synthetic cannabis-induced mania, Case Reports Psychiatry, 2015:310930. doi: 10.1155/2015/310930.

Van Rossum, I., Boomsma, M., Tenback, D., Reed, C. y Van Os, J.; EMBLEM Advisory Board. (2009). Does cannabis use affect treatment outcome in bipolar disorder? A longitudinal analysis. The Journal of Nervous and Mental Disease.197:3540. doi: 10.1097/NMD.0b013e31819292a6.

Weinstock, L.M., Gaudiano, B.A., Wenze, S.J., Epstein-Lubow, G. y Miller, I.W. (2016). Demographic and clinical characteristics associated with comorbid cannabis use disorders (CUDs) in hospitalized patients with bipolar I disorder. Comprehensive Psychiatry, 65:57-62. doi: 10.1016/j.comppsych.2015.10.003.

Zorrilla, I., Aguado, J., Haro, J.M., Barbeito, S., López, S., Ortiz, A., López, P. y Gonzalez-Pinto, A. (2015). Cannabis and bipolar disorder: does quitting cannabis use during manic/mixed episode improve clinical/functional outcomes? Acta Psychiatrica Scandinavica, 131:100-10. doi: 10.1111/acps.12366. 


\section{ANEXOS}

\section{ABREVIATURAS}

ASI: Addiction Severity Index.

CB1 y CB2: receptores cannabinoides tipo 1 y 2.

CGI-BP: Clinical Global Impression Scale for use in bipolar illness.

CIE: Clasificación Internacional de Enfermedades.

DSM: Diagnostic and Statistical Manual of Mental Disorders.

ECA: Epidemiologic Catchment Area Project.

GAF: Global Assessment of Functioning Scale.

HAMD: Hamilton Depression Rating Scale.

IDS-C: Inventory of Depression Scale.

MINI: Mini International Neuropsychiatric Interview.

NESARC: National Epidemiologic Survey on Alcohol and Related Conditions.

NCS: National Comorbidity Survey.

PANSS: The Positive and Negative Syndrome Scale

PAS: Premorbid Academic Functioning

SCID: Structured Clinical Interview for DSM Disorders.

TB: Trastorno bipolar.

THC: delta-9-tetrahidrocannabinol.

TM: Trastornos mentales.

TUC: Trastorno por uso de cannabis.

TUS: Trastorno por uso de sustancias.

YMRS: Young Mania Rating Scale. 


\section{TRASTORNO BIPOLAR}

\section{Tabla 1. Criterios diagnósticos DSM-5 de los trastornos bipolares. (Tomado de Manual Diagnóstico y} Estadístico de los Trastornos Mentales DSM-5. 2015)

\section{Criterios diagnósticos:}

Para un diagnóstico de trastorno bipolar es necesario que se cumplan los criterios siguientes para un episodio maniaco. Antes o después del episodio maniaco pueden haber existido episodios hipomaniacos o episodios de depresión mayor.

\section{Episodio maniaco:}

a) Periodo definido de estado de ánimo anormal y persistentemente elevado, expansivo o irritable, y un aumento anormal y persistente de la actividad o la energía, que dura como mínimo una semana y está presente la mayor parte del día, casi todos los días (o cualquier duración si se necesita hospitalización)

b) Durante el periodo de alteración del estado de ánimo y aumento de la energía o la actividad, existen 30 más (4 si el estado es sólo irritable) de los siguientes en un grado significativo y representan un cambio notorio del comportamiento habitual:

1. Aumento de la autoestima o sentimiento de grandeza.

2. Disminución de la necesidad de dormir.

3. Más hablador de lo habitual o presión para mantener la conversación.

4. Fuga de ideas o experiencia subjetiva de que los pensamientos van a gran velocidad.

5. Facilidad de distracción.

6. Aumento de la actividad dirigida a un objeto o agitación psicomotora.

7. Participación excesiva en actividades que tienen muchas posibilidades de consecuencias dolorosas (Ejemplos.: dedicarse de forma desenfrenada a las compras, inversiones de dinero imprudentes)

Episodio hipomaniaco:

a) Periodo bien definido de estado de ánimo anormal y persistentemente elevado, expansivo o irritable, y un aumento anormal y persistente de la actividad o la energía, que dura como mínimo 4 días consecutivos y está presente la mayor parte del día, casi todos los días.

b) Durante el periodo de alteración del estado de ánimo y aumento de la energía o la actividad, existen 3 o más (4 si el estado es sólo irritable) de los siguientes en un grado significativo y representan un cambio notorio del comportamiento habitual:

1. Aumento de la autoestima o sentimiento de grandeza.

2. Disminución de la necesidad de dormir.

3. Más hablador de lo habitual o presión para mantener la conversación.

4. Fuga de ideas o experiencia subjetiva de que los pensamientos van a gran velocidad.

5. Facilidad de distracción.

6. Aumento de la actividad dirigida a un objeto o agitación psicomotora.

7. Participación excesiva en actividades que tienen muchas posibilidades de consecuencias dolorosas (Ejemplos.: dedicarse de forma desenfrenada a las compras, inversiones de dinero imprudentes)

c) El episodio se asocia a un cambio inequívoco del funcionamiento que no es característico del individuo cuando no presenta síntomas.

d) La alteración del estado de ánimo y el cambio en el funcionamiento son observables por parte de otras personas.

e) El episodio no es suficientemente grave para causar una alteración importante del funcionamiento social o laboral, o necesitar hospitalización. Si existen características psicóticas, el episodio es por definición, maníaco.

f) El episodio no se puede atribuir a los efectos fisiológicos de una sustancia. 
Episodio de depresión mayor:

a) 5 o más de los síntomas siguientes han estado presentes durante el mismo periodo de 2 semanas y representan un cambio del funcionamiento anterior; al menos uno de los síntomas es estado de ánimo deprimido o pérdida de interés o de placer.

1. Estado de ánimo deprimido la mayor parte del día, casi todos los días, (En niños y adolescentes el estado de ánimo puede ser irritable).

2. Disminución importante del interés o el placer por todas o casi todas las actividades la mayor parte del día, casi todos los días.

3. Pérdida importante de peso sin hacer dieta o aumento de peso, o disminución o aumento del apetito casi todos los días.

4. Insomnio o hipersomnia casi todos los días.

5. Agitación o retraso psicomotor casi todos los días.

6. Fatiga o pérdida de la energía casi todos los días.

7. Sentimientos de inutilidad o de culpabilidad excesiva o inapropiada (que puede ser delirante) casi todos los días.

8. Disminución de la capacidad para pensar o concentrarse, o de tomar decisiones, casi todos los días.

9. Pensamientos de muerte recurrentes, ideas suicidas recurrentes sin un plan determinado, intento de suicidio o un plan específico para llevarlo a cabo.

b) Los síntomas causan malestar clínicamente significativo o deterioro en lo social, laboral u otras áreas importantes del funcionamiento.

El episodio no se puede atribuir a los efectos fisiológicos de una sustancia o de otra afección médica.

Tabla 2. Trastorno ciclotímico. (Tomado de Manual Diagnóstico y Estadístico de los Trastornos Mentales DSM-5. 2015)

a) Durante 2 años como mínimo (al menos 1 en niños y adolescentes) han existido numerosos periodos con síntomas hipomaníacos que no cumplen los criterios para un episodio hipomaníaco y numerosos períodos con síntomas depresivos que no cumplen los criterios para un episodio de depresión mayor.

b) Durante el periodo de 2 años (1 en niños y adolescentes), los periodos hipomaníacos y depresivos han estado presentes al menos la mitad del tiempo y el individuo no ha presentado síntomas durante más de 2 meses seguidos.

c) Nunca se han cumplido los criterios para un episodio de depresión mayor, maníaco o hipomaníaco.

d) Los síntomas del criterio a no se explican mejor por un trastorno esquizoafectivo, esquizofrenia, un trastorno esquizofreniforme, un trastorno de ideas delirantes, u otro trastorno del espectro de la esquizofrenia y otros trastornos psicóticos especificados o no especificados.

e) Los síntomas no se pueden atribuir a los efectos fisiológicos de una sustancia o a otra afección médica.

f) Los síntomas causan malestar clínicamente significativo o deterioro en lo social, laboral u otras áreas importantes del funcionamiento. 
Tabla 3. Clasificación CIE-10. (Tomada de Trastornos Mentales y del Comportamiento. Décima Revisión de la Clasificación Internacional de las Enfermedades. 1992)

\section{F30-39 Trastornos del humor (afectivos):}

- F31 Trastorno bipolar:

F31.0. Trastorno bipolar, episodio actual hipomaníaco.

F31.1. Trastorno bipolar, episodio actual maníaco sin síntomas psicóticos.

F31.2. Trastorno bipolar, episodio actual maníaco con síntomas psicóticos.

F31.3. Trastorno bipolar, episodio actual depresivo leve o moderado.

F31.4. Trastorno bipolar, episodio actual depresivo grave sin síntomas psicóticos.

F31.5. Trastorno bipolar, episodio actual depresivo grave con síntomas psicóticos.

F31.6. Trastorno bipolar, episodio actual mixto.

F31.7. Trastorno bipolar, actualmente en remisión.

F31.8. Otros trastornos bipolares.

F31.9. Trastorno bipolar sin especificación.

Tabla 4. Principales diferencias y similitudes entre el DSM-5 y la CIE-11. Modificado de Dios et al. (2014).

\begin{tabular}{|l|l|l}
\hline Trastornos bipolares & DSM-5 & CIE-11
\end{tabular}

Convergencias:

- Se incluye como requisito el aumento de actividad o energía como criterio diagnóstico de hipomanía.

- El TB tipo II, el TB tipo I episodio maniaco único y la manía inducida por tratamientos son categorías específicas.

\begin{tabular}{l|l|l}
\hline Divergencias: & \\
\hline$-\quad$ Episodio mixto & Se convierte en especificador. & Se mantiene. \\
& Se operativizan criterios ("otros & No se contempla. \\
& TB"). & \\
\hline
\end{tabular}




\section{CANNABIS}
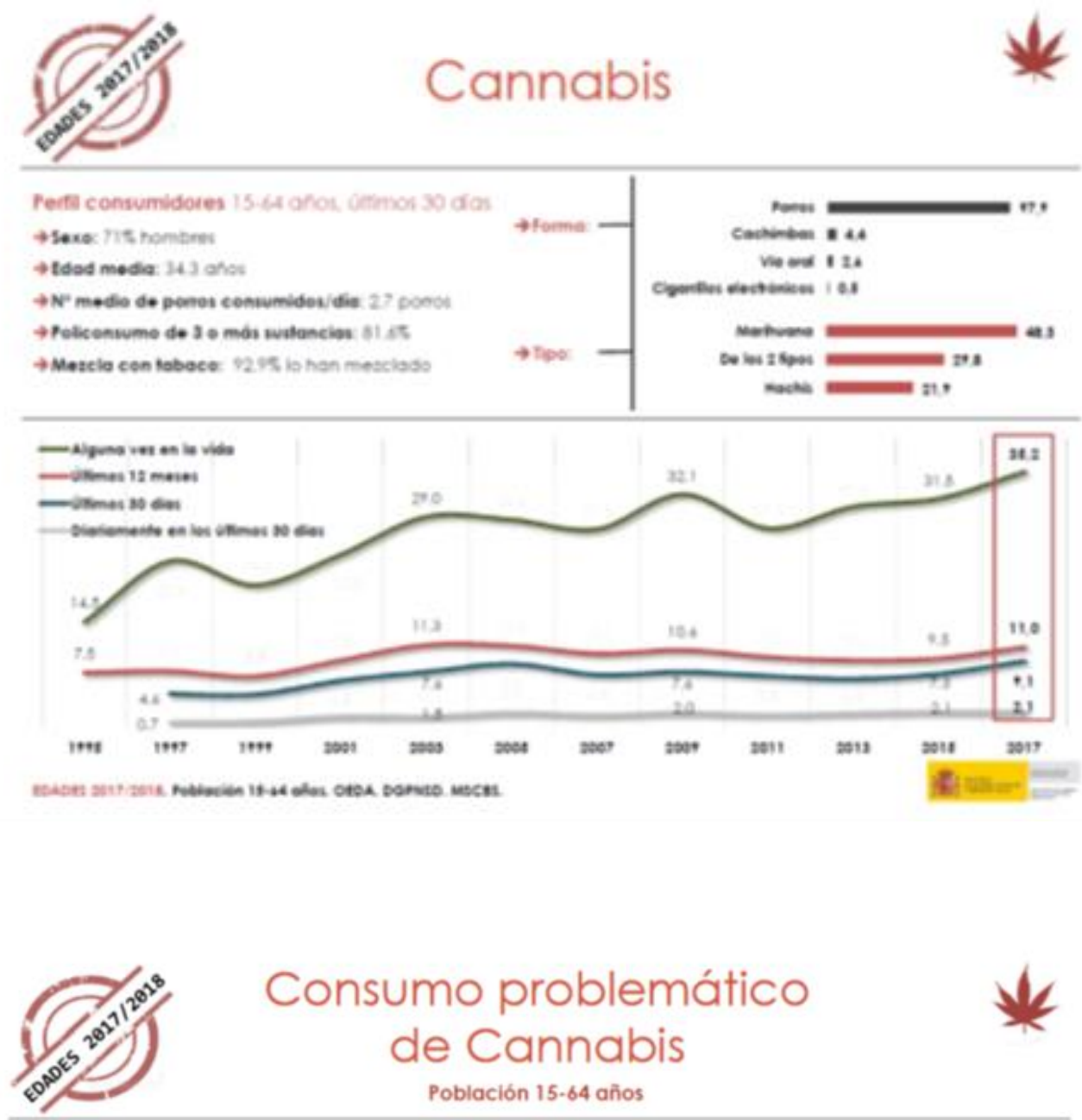

Perfil consumidores problemáticos

ültimos 12 meses

$\rightarrow$ Sexo: 77,75 hombres

497.000

$\rightarrow$ Edad media: 33.3 anos

personas hacen

$1,6 \%$ de toda la población

$\rightarrow N^{*}$ medio de porros consumidos/dia*t: 3.6 porros

consumo problemático

$\rightarrow$ Policonsumo de $3 \circ$ más sustancias: 90,45 de cannobis

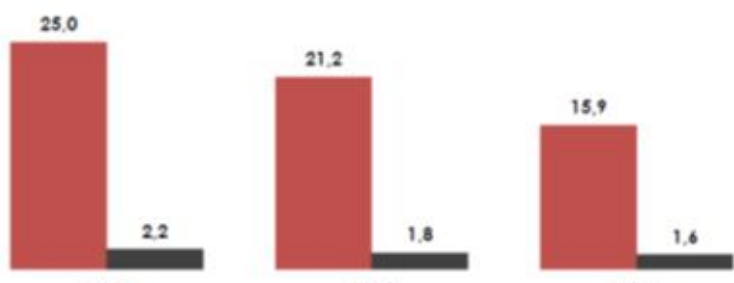

2013

" $\%$ entre la población (15-64 aȟos) que han consumido cannabis*

$=$ K entre toda la población (15-64 ahios)

Nota: El consumo problemotico se basa en la mcala C.NT (Connabis Abuse Screening tert).

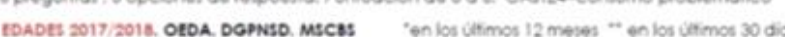

Figuras 1 y 2. Observatorio Europeo de las drogas y las toxicomanías, (2017). 
Tabla 5. Criterios diagnósticos DSM-5 de la intoxicación por cannabis. (Tomado de Manual Diagnóstico y Estadístico de los Trastornos Mentales DSM-5. 2015)

\section{Criterios diagnósticos:}

a) Consumo reciente de cannabis.

b) Comportamiento problemático o cambios psicológicos clínicamente significativos. (Ejemplos: descoordinación motora, euforia, ansiedad).

c) 2 o más de los signos o síntomas siguientes que aparecen en el plazo de 2 horas tras el consumo de cannabis:

- Inyección conjuntival.

- Aumento del apetito.

- Boca seca.

- Taquicardia.

d) Los signos o síntomas no se pueden atribuir a ninguna otra afección médica y no se explican mejor por otro trastorno mental, incluido una intoxicación con otra sustancia.

Especificar si con alteraciones de la percepción.

Tabla 6. Efectos agudos del consumo de cannabis. Modificado del Informe de la Comisión Clínica de la Delegación del Gobierno para el Plan Nacional sobre Drogas. Informe sobre cannabis. 2006.

\begin{tabular}{|l|l|}
\hline Efectos centrales & Consecuencia clínica \\
\hline Psicológico & Euforia, bienestar, relajación \\
\hline Cognitivos & $\begin{array}{l}\text { Alteración de la memoria a corto plazo, dificultad de } \\
\text { concentración }\end{array}$ \\
\hline Efectos sobre rendimiento psicomotor & $\begin{array}{l}\text { Empeoramiento tiempo de reacción y atención. Incoordinación } \\
\text { motora }\end{array}$ \\
\hline Efectos sobre la conducción de vehículos & Empeoramiento de conducción. Mayor riesgo de accidentes. \\
\hline Efectos sobre el sueño & Somnolencia \\
\hline Efectos sobre el apetito & Aumento de apetito \\
\hline Analgesia & Disminución del dolor \\
\hline Efectos sistémicos & Consecuencia clínica \\
\hline Efectos cardiovasculares & $\begin{array}{l}\text { Aumento de la frecuencia cardíaca. Aumento de presión } \\
\text { arterial. Aumento gasto cardíaco }\end{array}$ \\
\hline Efectos respiratorios & $\begin{array}{l}\text { Ligera broncodilatación. Exposición al humo y sustancias } \\
\text { cancerígenas del tabaco }\end{array}$ \\
\hline Efectos oculares & Enrojecimiento conjuntival. Reducción presión intraocular \\
\hline Efectos gastrointestinales & Sequedad de boca. Antiemesis \\
\hline Efectos sobre músculo estriado & Relajación muscular \\
\hline Efectos inmunológicos & Alteración de la inmunidad celular \\
\hline Efectos endocrinos & Disminución hormonas sexuales. Aumento de la prolactina \\
\hline Efectos en el embarazo y lactancia & $\begin{array}{l}\text { Mayor riesgo de bajo peso al nacer. Paso de cannabinoides a } \\
\text { la leche }\end{array}$ \\
\hline
\end{tabular}


Tabla 7. Criterios diagnósticos DSM-5 de la abstinencia de cannabis. (Tomado de Manual Diagnóstico y Estadístico de los Trastornos Mentales DSM-5. 2015)

\section{Criterios diagnósticos:}

a) Cese brusco del consumo de cannabis, que ha sido intenso y prolongado.

b) Aparición de 3 o más de los signos y síntomas siguientes aproximadamente en el plazo de una semana tras el criterio a.

1. Irritabilidad, rabia o agresividad.

2. Nerviosismo 0 ansiedad.

3. Dificultades para dormir.

4. Pérdida de apetito o de peso.

5. Intranquilidad.

6. Estado de ánimo deprimido.

7. Por lo menos uno de los síntomas físicos siguientes que provoca una incomodidad significativa: dolor abdominal, espasmos y temblores, sudoración, fiebre, escalofríos o cefalea.

c) Los signos o síntomas del Criterio b provocan un malestar clínicamente significativo o deterioro en lo social, laboral u otras áreas importantes del funcionamiento.

d) Los signos o síntomas no se pueden atribuir a ninguna otra afección médica y no se explican mejor por otro trastorno mental, incluidas una intoxicación o abstinencia de otra sustancia.

Tabla 8. Criterios diagnósticos DSM-5 del trastorno por consumo de cannabis. (Tomado de Manual Diagnóstico y Estadístico de los Trastornos Mentales DSM-5. 2015)

\section{Criterios diagnósticos:}

a) Patrón problemático de consumo de cannabis que provoca un deterioro o malestar clínicamente significativo y que se manifiesta al menor por 2 de los siguientes hechos en un plazo de 12 meses:

1. Se consume cannabis con frecuencia en cantidades superiores o durante un tiempo más prolongado del previsto.

2. Existe un deseo persistente o esfuerzos fracasados de abandonar o controlar el consumo de cannabis.

3. Se invierte mucho tiempo en las actividades necesarias para conseguir cannabis, consumirlo 0 recuperarse de sus efectos.

4. Ansias o un poderoso deseo o necesidad de consumir cannabis.

5. Consumo recurrente de cannabis que lleva al incumplimiento de los deberes fundamentales en el trabajo, la escuela o el hogar.

6. Consumo continuado de cannabis a pesar de sufrir problemas sociales 0 interpersonales persistentes o recurrentes, provocados o exacerbados por los efectos del mismo.

7. El consumo de cannabis provoca el abandono o la reducción de importantes actividades sociales, profesionales o de ocio.

8. Consumo recurrente de cannabis en situaciones en las que provoca un riesgo físico.

9. Se continúa con el consumo de cannabis a pesar de saber que se sufre un problema físico o psicológico persistente o recurrente probablemente causado o exacerbado por el mismo.

10. Tolerancia, definida por una necesidad de cantidades cada vez mayores de cannabis para conseguir la intoxicación o el efecto deseado o por un efecto notablemente reducido tras el consumo continuado de la misma cantidad de cannabis.

11. Abstinencia, manifestada por la presencia del síndrome de abstinencia característico del cannabis 0 se consume cannabis para aliviar o evitar los síntomas de la abstinencia.

Especificar la gravedad actual: Leve (presencia de 2-3 síntomas), Moderado (presencia de 4-5 síntomas), Grave (presencia de 6 o más síntomas). 
Tabla 9. F1x.1 Consumo perjudicial. (Tomada de Trastornos Mentales y del Comportamiento. Décima Revisión de la Clasificación Internacional de las Enfermedades. 1992)

Se trata de una forma de consumo que está afectando ya a la salud física (como en los casos de hepatitis por administración de sustancias psicotropas por vía parenteral) o mental, como, por ejemplo, los episodios de trastornos depresivos secundarios al consumo excesivo de alcohol.

El diagnóstico requiere que se haya afectado la salud mental o física del que consume la sustancia.

Las formas perjudiciales de consumo son a menudo criticadas por terceros y suelen dar lugar a consecuencias sociales adversas de varios tipos. El hecho de que una forma de consumo o una sustancia en particular sean reprobados por terceros o por el entorno en general, no es por sí mismo indicativo de un consumo perjudicial, como tampoco lo es sólo el hecho de haber podido derivar en alguna consecuencia social negativa tales como detención o ruptura matrimonial.

\section{Tabla 10. F1x.2 Síndrome de dependencia. (Tomada de Trastornos Mentales y del Comportamiento. Décima Revisión de la Clasificación Internacional de las Enfermedades. 1992)}

El diagnóstico de dependencia sólo debe hacerse si durante en algún momento en los doce meses previos 0 de un modo continuo han estado presentes tres o más de tres de los rasgos siguientes:

a) Deseo intenso o vivencia de una compulsión a consumir una sustancia.

b) Disminución de la capacidad para controlar el consumo de una sustancia o alcohol, unas veces para controlar el comienzo del consumo y otras para poder terminarlo para controlar la cantidad consumida.

c) Síntomas somáticos de un síndrome de abstinencia (ver F1x.4, F1x.3) cuando el consumo de la sustancia se reduzca o cese, cuando se confirme por: el síndrome de abstinencia característico de la sustancia; 0 el consumo de la misma sustancia (o de otra muy próxima) con la intención de aliviar o evitar síntomas de abstinencia.

d) Tolerancia, de tal manera que se requiere un aumento progresivo de la dosis de la sustancia para conseguir los mismos efectos que originalmente producían dosis más bajas (son ejemplos claros los de la dependencia al alcohol y a los opiáceos, en las que hay individuos que pueden llegar a ingerir dosis suficientes para incapacitar o provocar la muerte a personas en las que no está presente una tolerancia).

e) Abandono progresivo de otras fuentes de placer o diversiones, a causa del consumo de la sustancia, aumento del tiempo necesario para obtener o ingerir la sustancia o para recuperarse de sus efectos.

h) Persistencia en el consumo de la sustancia a pesar de sus evidentes consecuencias perjudiciales, tal y como daños hepáticos por consumo excesivo de alcohol, estados de ánimo depresivos consecutivos a períodos de consumo elevado de una sustancia o deterioro cognitivo secundario al consumo de la sustancia. Debe investigarse a fondo si la persona que consuma la sustancia es consciente, o puede llegar a serlo de la naturaleza y gravedad de los perjuicios. 